\title{
Comparison principles and Lipschitz regularity for some nonlinear degenerate elliptic equations
}

\author{
YanYan Li ${ }^{*}$ Luc Nguyen ${ }^{\dagger}$ Bo Wang ${ }^{\ddagger}$
}

\begin{abstract}
We establish interior Lipschitz regularity for continuous viscosity solutions of fully nonlinear, conformally invariant, degenerate elliptic equations. As a by-product of our method, we also prove a weak form of the strong comparison principle, which we refer to as the principle of propagation of touching points, for operators of the form $\nabla^{2} \psi+L(x, \psi, \nabla \psi)$ which are non-decreasing in $\psi$.

Key words: Lipschitz regularity; comparison principle; propagation of touching points; degenerate elliptic; conformal invariance.
\end{abstract}

MSC2010: 35J60 35J70 35B51 35B65 35D40 53C21 58J70

\section{Introduction}

The main goal of this paper is to prove interior Lipschitz regularity for continuous viscosity solutions of fully nonlinear, conformally invariant, degenerate elliptic equations arising from conformal geometry.

Let $\mathbb{R}^{n}$ denote the Euclidean space of dimension $n$,

$\Gamma \subset \mathbb{R}^{n}$ be an open convex symmetric cone with vertex at the origin,

*School of Mathematical Sciences, Beijing Normal University, Beijing 100875, China and Department of Mathematics, Rutgers University, 110 Frelinghuysen Rd, Piscataway, NJ 08854, USA. Email: yyli@math.rutgers.edu.

${ }^{\dagger}$ Mathematical Insitute and St Edmund Hall, University of Oxford, Andrew Wiles Building, Radcliffe Observatory Quarter, Woodstock Road, Oxford OX2 6GG, UK. Email: luc.nguyen@maths.ox.ac.uk.

${ }^{\ddagger}$ School of Mathematics and Statistics, Beijing Institute of Technology, Beijing 100081, China. Email: wangbo89630@bit.edu.cn. 
satisfying

$$
\Gamma \supset\left\{\lambda \in \mathbb{R}^{n}: \lambda_{i}>0, i=1, \cdots, n\right\} .
$$

Let $f \in C^{1}(\Gamma) \cap C^{0}(\bar{\Gamma})$ be a symmetric function satisfying

$$
f=0 \text { on } \partial \Gamma \text { and } f>0, \quad \frac{\partial f}{\partial \lambda_{i}}>0 \text { in } \Gamma \text { for } i=1, \ldots, n .
$$

In the above, the symmetricity of $(f, \Gamma)$ is understood in the sense that if $\lambda \in \Gamma$, then $\tilde{\lambda} \in \Gamma$ and $f(\tilde{\lambda})=f(\lambda)$ for any permutation $\tilde{\lambda}$ of $\lambda$.

For a function $u$ defined on a Euclidean domain, let $A^{u}$ denote its conformal Hessian matrix, i.e.

$$
A^{u}:=-\frac{2}{n-2} u^{-\frac{n+2}{n-2}} \nabla^{2} u+\frac{2 n}{(n-2)^{2}} u^{-\frac{2 n}{n-2}} \nabla u \otimes \nabla u-\frac{2}{(n-2)^{2}} u^{-\frac{2 n}{n-2}}|\nabla u|^{2} I,
$$

where here and below $I$ denotes the $n \times n$ identity matrix, and, for $p, q \in \mathbb{R}^{n}$, $p \otimes q$ denotes the $n \times n$ matrix with entries $(p \otimes q)_{i j}=p_{i} q_{j}$. Let $\lambda\left(A^{u}\right)$ denote the eigenvalues of $A^{u}$.

In recent years, there has been a growing literature on the following two equations:

$$
f\left(\lambda\left(A^{u}\right)\right)=1, \quad u>0 \text { and } \quad \lambda\left(A^{u}\right) \in \Gamma
$$

and

$$
\lambda\left(A^{u}\right) \in \partial \Gamma, \quad \text { and } \quad u>0 .
$$

Note that equation (5) is equivalent to

$$
f\left(\lambda\left(A^{u}\right)\right)=0, \quad u>0 \quad \text { and } \quad \lambda\left(A^{u}\right) \in \bar{\Gamma} .
$$

Equation (44) and (5) are second order fully nonlinear elliptic and degenerate elliptic equations, respectively. Fully nonlinear elliptic equations involving $f\left(\lambda\left(\nabla^{2} u\right)\right)$ was investigated in the classic paper [8].

The equations (4) and (5) arose from conformal geometry. On a Riemannian manifold $(M, g)$ of dimension $n \geq 3$, consider the Schouten tensor

$$
A_{g}=\frac{1}{n-2}\left(\operatorname{Ric}_{g}-\frac{1}{2(n-1)} R_{g} g\right)
$$

where $\mathrm{Ric}_{g}$ and $R_{g}$ denote, respectively, the Ricci tensor and the scalar curvature. Let $\lambda\left(A_{g}\right)=\left(\lambda_{1}, \cdots, \lambda_{n}\right)$ denote the eigenvalues of $A_{g}$ with respect to $g$. It is well known that, in a conformal change of the metric, the "main contribution" 
to the curvature tensor is captured in the change of the Schouten tensor. One is thus naturally led to study, in the hope of finding some sort of "best metric" in a conformal class of metrics, the problem (see e.g. [9, 42])

$$
f\left(\lambda\left(A_{u^{\frac{4}{n-2}} g}\right)\right)=1, \quad u>0, \quad \text { and } \quad \lambda\left(A_{u^{\frac{4}{n-2} g}}\right) \in \Gamma \text { on } M .
$$

This problem is sometimes referred to in the literature as a fully nonlinear version of the Yamabe problem. When $M$ is a Euclidean domain and $g=g_{\text {flat }}$ is the flat metric, equation (6) is exactly equation (41). Furthermore, both equation (41) and equation (5) appear naturally in the study of blow-up sequences of solutions of (6) on manifolds.

Important examples of $(f, \Gamma)$ are $(f, \Gamma)=\left(\sigma_{k}^{\frac{1}{k}}, \Gamma_{k}\right), 1 \leq k \leq n$, where $\sigma_{k}(\lambda):=$ $\sum_{1 \leq i_{1}<\cdots<i_{k} \leq n} \lambda_{i_{1}} \cdots \lambda_{i_{k}}$ is the $k$-th elementary symmetric function, and $\Gamma_{k}$ is the connected component of $\left\{\lambda \in \mathbb{R}^{n}: \sigma_{k}(\lambda)>0\right\}$ containing the positive cone $\left\{\lambda \in \mathbb{R}^{n}\right.$ : $\left.\lambda_{i}>0, i=1, \cdots, n\right\}$. When $(f, \Gamma)=\left(\sigma_{1}, \Gamma_{1}\right)$, (6) is the classical Yamabe problem in the so-called positive case.

In this paper, we establish the following regularity result for continuous viscosity solutions of (5). See [33, Definition 1.1] and Definition 1.3 below for the definition of viscosity solutions.

Theorem 1.1 (Local Lipschitz regularity). For $n \geq 3$, let $\Omega$ be an open subset of $\mathbb{R}^{n}$, and $\Gamma$ satisfy (1) and (2). Assume that $u$ is a continuous viscosity solution of (5) in $\Omega$. Then $u \in C_{\text {loc }}^{0,1}(\Omega)$.

Remark 1.2. As a consequence of Theorem 1.1, several previously known results for Lipschitz continuous solutions of (5) hold for continuous solutions. This includes the Liouville-type Theorem 1.4, the symmetry results Theorem 1.18 and Theorem 1.23 in [33]; the Bôcher-type Theorems 1.2 and 1.3, the Harnack-type Theorem 1.5, and the asymptotic behavior results Corollary 1.7 and Theorem 1.8 in 34 .

Although there have been many works on a priori estimates for solutions to (41) and (5) and closely related issues (see e.g. [9, 10, 14, 15, 16, 17, 18, 24, 25, 31, 32, 33, 34, 35, 38, 39, 41, 43, 44]), our theorem above appears to be the first regularity result for viscosity solutions in this context.

The regularity obtained in Theorem 1.1] is in a sense sharp: In [38], Nadirashvili and Vlădut showed that, for any $\epsilon \in(0,1)$, there exists a solution to a uniformly elliptic and conformally invariant equation in a ball $B \subset \mathbb{R}^{5}$ which belongs to $C^{1, \epsilon}(B) \backslash C^{1, \epsilon+}(B)$. 
It is sometimes more convenient to write $u=e^{-\frac{n-2}{2} \psi}$ or $u=w^{-\frac{n-2}{2}}$. An easy computation gives $A^{u}=A_{w}=e^{2 \psi} A[\psi]$ where

$$
\begin{aligned}
A_{w} & =w \nabla^{2} w-\frac{1}{2}|\nabla w|^{2} I, \\
A[\psi] & =\nabla^{2} \psi+\nabla \psi \otimes \nabla \psi-\frac{1}{2}|\nabla \psi|^{2} I .
\end{aligned}
$$

In addition to Theorem 1.1, we also study the Dirichlet boundary value problem for a class of degenerate elliptic equations which includes the conformal operator $A[\psi]$. Consider operators of the form

$$
F[\psi]=\nabla^{2} \psi+\alpha \nabla \psi \otimes \nabla \psi-\beta|\nabla \psi|^{2} I
$$

where $\alpha$ and $\beta$ are constant, and the equation

$$
F[\psi] \in \partial U
$$

where $U$ is a non-empty open subset of $\mathcal{S}^{n \times n}$ satisfying a degenerate ellipticity condition:

$$
\text { if } A \in U, B \in \mathcal{S}^{n \times n} \text { and } B>0 \text {, then } A+B \in U \text {. }
$$

(Note that (8) implies that $\partial U$ is Lipschitz.)

In the context of Theorem 1.1, $U$ is the set of symmetric matrices whose eigenvalues belong to $\Gamma$, as equation (5) can be written equivalently as $A^{u} \in \partial U$. We note for future use that our results below apply also to the setting of fully nonlinear Yamabe problem of "negative type" by considering the set $U$ of symmetric matrices whose eigenvalues belong to $\mathbb{R}^{n} \backslash(-\bar{\Gamma})$, where

$$
-\bar{\Gamma}=\left\{\lambda \in \mathbb{R}^{n}:-\lambda \in \bar{\Gamma}\right\} .
$$

In both cases, (8) holds thanks to (11) and (2).

For any set $S \subset \mathbb{R}^{n}$, we use $\operatorname{USC}(S)$ to denote the set of functions $\psi: S \rightarrow$ $\mathbb{R} \cup\{-\infty\}, \psi \neq \equiv-\infty$ in $S$, satisfying

$$
\limsup _{x \rightarrow \bar{x}} \psi(x) \leq \psi(\bar{x}), \quad \forall \bar{x} \in S .
$$

Similarly, we use $\operatorname{LSC}(S)$ to denote the set of functions $\psi: S \rightarrow \mathbb{R} \cup\{+\infty\}, \psi \not \equiv+\infty$ in $S$, satisfying

$$
\liminf _{x \rightarrow \bar{x}} \psi(x) \geq \psi(\bar{x}), \quad \forall \bar{x} \in S .
$$

We now give the definition of viscosity subsolutions, supersolutions and solutions to the degenerate elliptic equation $F[\psi] \in \partial U$. 
Definition 1.3. Let $\Omega \subset \mathbb{R}^{n}, n \geq 2$, be an open set, and $U$ be a non-empty open subset of $\mathcal{S}^{n \times n}$ satisfying (8). For a function $\psi$ in $U S C(\Omega)(L S C(\Omega))$, we say that

$$
F[\psi] \in \bar{U} \quad\left(F[\psi] \in \mathcal{S}^{n \times n} \backslash U\right) \quad \text { in } \Omega \quad \text { in the viscosity sense }
$$

if for any $x_{0} \in \Omega, \varphi \in C^{2}(\Omega),(\psi-\varphi)\left(x_{0}\right)=0$ and

$$
\psi-\varphi \leq 0 \quad(\psi-\varphi \geq 0), \quad \text { near } x_{0}
$$

there holds

$$
F[\varphi]\left(x_{0}\right) \in \bar{U} \quad\left(F[\varphi]\left(x_{0}\right) \in \mathcal{S}^{n \times n} \backslash U\right) .
$$

We say that a function $\psi \in C^{0}(\Omega)$ satisfies

$$
F[\psi] \in \partial U \text { in the viscosity sense }
$$

in $\Omega$ if $F[\psi]$ belongs to both $\bar{U}$ and $\mathcal{S}^{n \times n} \backslash U$ in $\Omega$ in the viscosity sense.

When $F[\psi] \in \bar{U} \quad\left(F[\psi] \in \mathcal{S}^{n \times n} \backslash U\right)$ in $\Omega$ in the viscosity sense, we also say interchangeably that $\psi$ is a viscosity subsolution (supersolution) to (9) in $\Omega$.

Our next result is a uniqueness statement for (9) when $U$ satisfies

$$
A \in U \text { and } c>0 \Rightarrow c A \in U \text {. }
$$

Theorem 1.4 (Uniqueness for the Dirichlet Problem). Let $\Omega \subset \mathbb{R}^{n}(n \geq 2)$ be a non-empty bounded open set, and $U$ be a non-empty open subset of $\mathcal{S}^{n \times n}$ satisfying (8) and (10). Assume that $F$ is of the form (7). Then, for any $\varphi \in C^{0}(\partial \Omega)$, there exists at most one solution $\psi \in C^{0}(\bar{\Omega})$ of (9) satisfying $\psi=\varphi$ on $\partial \Omega$.

We also prove the following existence theorem using Perron's method (see [21]).

Theorem 1.5 (Existence by sub- and supersolution method). Let $\Omega$ and $(F, U)$ be as in Theorem 1.4. Let $w \in L S C(\bar{\Omega})$ and $v \in U S C(\bar{\Omega})$ be respectively supersolution and subsolution of (9) in $\Omega$ such that $w \geq v$ in $\Omega$ and $w=v$ on $\partial \Omega$. Then there exists a viscosity solution $u \in C^{0}(\bar{\Omega})$ of $(9)$ in $\Omega$ satisfying

$$
\begin{array}{ll}
v \leq u \leq w & \text { in } \bar{\Omega}, \\
u=w=v & \text { on } \partial \Omega .
\end{array}
$$

One main ingredient of the proofs of Theorems 1.1, 1.4 and 1.5 is a comparison principle. In recent years, comparison principles (for viscosity solutions) have been very successfully applied to derive estimates and symmetry properties for solutions 
to (both degenerate and non-degenerate elliptic) equations in conformal geometry; see 33 . and the references therein. Our paper can be viewed as a continuation in this line of work.

In fact, we will establish a variant of the comparison principle for more general operators of the form

$$
F[\psi]=\nabla^{2} \psi+\alpha(\cdot, \psi) \nabla \psi \otimes \nabla \psi-\beta(\cdot, \psi)|\nabla \psi|^{2} I
$$

where $\alpha, \beta: \Omega \times \mathbb{R} \rightarrow \mathbb{R}$ and $\Omega$ is an open subset of $\mathbb{R}^{n}$. Throughout the paper, we will assume that

the function $L(x, s, p):=\alpha(x, s) p \otimes p-\beta(x, s)|p|^{2} I$ is non-decreasing in $s$.

Note that this condition is consistent with both $A[\psi]$ and $\frac{1}{w} A_{w}$ defined above.

In the sequel, we say that the principle of propagation of touching points holds for $(F, U)$ if for any supersolution $w \in L S C(\bar{\Omega})$ and subsolution $v \in U S C(\bar{\Omega})$ of (9) in $\Omega$ one has

$$
w \geq v \text { in } \Omega \text { and } w>v \text { on } \partial \Omega \quad \Rightarrow \quad w>v \text { in } \Omega .
$$

(In other words, if $w \geq v$ in $\Omega$ then every non-empty connected component of the set $\{x \in \bar{\Omega}: w(x)=v(x)\}$ contains a point of $\partial \Omega$.) This principle can be viewed as a weak version of the strong comparison principle.

We say that the comparison principle holds for $(F, U)$ if for any supersolution $w \in L S C(\bar{\Omega})$ and subsolution $v \in U S C(\bar{\Omega})$ of $(9)$ in $\Omega$ one has

$$
w \geq v \text { on } \partial \Omega \quad \Rightarrow \quad w \geq v \text { in } \Omega .
$$

It should be noted that, for general degenerate elliptic equations, $w \geq v$ in $\Omega$ does not imply the dichotomy that $w>v$ or $w \equiv v$ in $\Omega$. (This is in contrast with the uniformly elliptic case.)

Remark 1.6. If $L(x, s, p)$ is independent of $s$, then the principle of propagation of touching points is equivalent to the comparison principle.

We prove that the principle of propagation of touching points holds when $(F, U)$ satisfies, in addition to (8), (10) and (12), the following structural conditions:

either $|\beta(x, s)|>\beta_{0}>0$ for some constant $\beta_{0}$

or $\quad$ both $\alpha$ and $\beta$ are constant. 
Theorem 1.7 (Principle of propagation of touching points). Let $F$ be of the form (11) where $\alpha, \beta \in C_{\text {loc }}^{0,1}(\bar{\Omega} \times \mathbb{R})$ satisfy (12) and (13). Let $\Omega \subset \mathbb{R}^{n}(n \geq 2)$ be a non-empty bounded open set, and $U$ be a non-empty open subset of $\mathcal{S}^{n \times n}$ satisfying (8) and (10). Assume that $w \in L S C(\bar{\Omega})$ and $v \in U S C(\bar{\Omega})$ are respectively a supersolution and a subsolution of (9) in $\Omega$.

(a) If $w \geq v$ in $\Omega$ and $w>v$ on $\partial \Omega$, then $w>v$ in $\Omega$.

(b) In case $\alpha$ and $\beta$ are constant, if $w \geq v$ on $\partial \Omega$, then $w \geq v$ in $\Omega$.

When $w$ and $v$ are locally Lipschitz and $F[\psi]=A[\psi]$, Theorem 1.7 was established in 33.

The proof of Theorem 1.7yields the propagation principle for an even larger class of operators; see Theorem 3.2 (where the assumption on the quadratic dependence of $F[\psi]$ on $\nabla \psi$ is somewhat relaxed to a super-linear dependence). One ingredient of the proof is a first variation result which, roughly speaking, allows one to perturb a given function $\psi$ to another function $\tilde{\psi}$ such that $F[\tilde{\psi}]$ are either "more inside" or "more outside" the set $U$ than $F[\psi]$ in a detailed controlled fashion. There are two delicate points of this process. On the one hand, one needs to ensure that the gain obtained is strong enough to counter-balance the error accrued in either regularization or handling the difficulties created by degenerate ellipticity. On the other hand, the whole process is carried out in such a way that it depends only on an upper bound and a lower bound of $\psi$, and not on $\nabla \psi$ or $\nabla^{2} \psi$. It is in this first variation argument that the assumptions that $\beta$ does not change sign and $L$ is non-decreasing are crucially used. See subsection 3.5 for examples which hint that these assumptions cannot simply be dropped.

Comparison principles for different classes of (degenerate) elliptic operators are available in the literature. See 1, 22, 3, 4, 5, 11, 12, 13, 19, 20, 21, 22, 23, 26, 27, [28, 29, 30, 40] and the references therein. Most of these works assumed a kind of "properness/non-degeneracy" of the operator with respect to the unknown $\psi$ (e.g. $L$ is decreasing with respect to $s$ ) which is not applicable to our setting (see condition (12)). In the present paper, we exploit instead some non-degeneracy with respect to the derivatives $\nabla \psi$ of the unknown.

It is natural to ask if the method we follow here can be tweaked together with the more familiar treatment for proper operators to treat a broader class of operators, but this goes beyond the scope of the present paper. We however note that (non-strict) properness of the operator is far from ensuring the validity of a comparison/propagation principle; see Proposition 3.11. 
Using results on removable singularities in [7], we obtain the following comparison principle on domains with singularities when $U$ satisfies in addition the condition

$$
U \subset\left\{M \in \mathcal{S}^{n \times n}: \operatorname{tr}(M)>0\right\},
$$

where $\operatorname{tr}(M)$ denotes the trace of $M$. For the proof, see Section 3 .

Corollary 1.8. Let $\Omega \subset \mathbb{R}^{n}(n \geq 2)$ be a bounded non-empty open set, $E \subset \Omega$ be a closed set with zero Newtonian capacity, and $(F, U)$ be as in Theorem 1.7 with constant $\alpha$ and $\beta$. If $w \in L S C(\bar{\Omega})$ and $v \in U S C(\bar{\Omega} \backslash E)$ satisfy

$$
\begin{aligned}
& w \text { is a supersolution to (9) in } \Omega, \\
& v \text { is a subsolution to (9) in } \Omega \backslash E,
\end{aligned}
$$

$w \geq v$ in $\Omega \backslash E$ and $w>v$ on $\partial \Omega$, and if

$$
\begin{array}{r}
\text { either } \sup _{\Omega \backslash E} v<+\infty, \\
\text { or } \alpha-n \beta<0,
\end{array}
$$

then $\inf _{\Omega \backslash E}(w-v)>0$.

Remark 1.9. It is interesting to identify the set $S_{k}$ of $(\alpha, \beta)$ for which one cannot drop the assumption that $v$ is bounded from above when $U$ is the set of symmetric matrices whose eigenvalues belong to $\Gamma_{k}$ with $2 \leq k \leq n$. Note that by the above result, $S_{k} \subset\{\alpha-n \beta \geq 0\}$. For $k=1$, equation (9) becomes $\Delta \psi+(\alpha-n \beta)|\nabla \psi|^{2}=0$, from which one can see that $S_{1} \subset\{\alpha-n \beta>0\}$. In fact, $S_{1}=\{\alpha-n \beta>0\}$. To see this, note that the functions $\psi_{\mu}(x)=\frac{1}{\alpha-n \beta} \ln \left(|x|^{2-n}+\mu\right)$ with $\mu \geq 0$ are solutions of (9) in $B_{1}(0) \backslash\{0\}$. In particular, $w=\psi_{1}$ is a supersolution of (9) in $B_{1}(0), v=\psi_{0}$ is a subsolution of (9) in $B_{1}(0) \backslash\{0\}, w \geq v$ in $B_{1}(0) \backslash\{0\}$, and $w>v$ on $\partial B_{1}(0)$, but $\inf _{B_{1}(0) \backslash\{0\}}(w-v)=0$.

When $F[\psi]$ is the conformal operator $A[\psi]$, Corollary 1.8 was proved by the first named author in [33] under the assumption that $E \subset \Omega$ containing at most finitely many points, $U=\left\{M \in \mathcal{S}^{n \times n}: \lambda(M) \in \Gamma\right\}, w \in C^{0,1}(\bar{\Omega})$ and $v \in C^{0,1}(\bar{\Omega} \backslash E)$.

A related issue of interest is whether the strong maximum principle and the Hopf lemma holds. It turns out that, in this degenerate elliptic setting, both fail for a large class of operators. See [7, 37] for further discussion.

Last but not least, the proof of Theorem 1.1 uses not only the comparison principle (Theorem [1.7) but also conformal invariance properties of $A[\psi]$ (i.e. of $A^{u}$ ). We 
remark that, for general $F$ and $U$, the comparison principle itself is far from ensuring (Lipschitz) regularity of viscosity solutions. See Section 5 for further discussion.

The rest of the paper is structured as follows. We start in Section 2 with some preliminaries about regularizations of semi-continuous functions by lower and upper envelops. In Section [3, we prove a generalization of Theorem 1.7 for more general operators and give counterexamples to highlight the importance of the conditions in the theorem. In Section 4, we prove the uniqueness Theorem 1.4 and the existence result Theorem 1.5. Finally, in Section 5, we prove the regularity result Theorem 1.1 together with some generalization.

\section{Preliminaries}

We briefly recall a well-known regularization of semi-continuous functions which will be used later in the paper.

Assume $n \geq 1$ and let $\Omega$ be an open bounded set in $\mathbb{R}^{n}$. For a function $v \in$ $U S C(\bar{\Omega})$ and $\epsilon>0$, we define the $\epsilon$-upper envelop of $v$ by

$$
v^{\epsilon}(x):=\max _{y \in \bar{\Omega}}\left\{v(y)-\frac{1}{\epsilon}|y-x|^{2}\right\}, \quad \forall x \in \bar{\Omega} .
$$

Likewise, for a function $w \in L S C(\bar{\Omega})$, its $\epsilon$-lower envelop is defined by

$$
w_{\epsilon}(x):=\min _{y \in \bar{\Omega}}\left\{w(y)+\frac{1}{\epsilon}|y-x|^{2}\right\}, \quad \forall x \in \bar{\Omega} .
$$

Although our definition of upper and lower envelops is slightly different from the definition in [6], all relevant properties established in [6, Lemma 5.2] remain valid with minor modification. We collect below some useful properties.

(i) $v^{\epsilon}, w_{\epsilon}$ belong to $C(\bar{\Omega})$, are monotonic in $\epsilon$ and

$$
v_{\epsilon} \rightarrow v, w_{\epsilon} \rightarrow w \text { pointwise as } \epsilon \rightarrow 0 \text {. }
$$

(ii) $v^{\epsilon}$ and $w_{\epsilon}$ are punctually second order differentiable (see e.g. [6] for a definition) almost everywhere in $\Omega$ and

$$
\nabla^{2} v^{\epsilon} \geq-\frac{2}{\epsilon} I, \quad \nabla^{2} w_{\epsilon} \leq \frac{2}{\epsilon} I, \quad \text { a.e. in } \Omega
$$


(iii) For any $x \in \Omega$, there exists $x^{*}=x^{*}(x) \in \bar{\Omega}$ such that

$$
v^{\epsilon}(x)=v\left(x^{*}\right)-\frac{1}{\epsilon}\left|x^{*}-x\right|^{2} \text { and }\left|x^{*}-x\right|^{2} \leq \epsilon\left(\max _{\bar{\Omega}} v-v(x)\right) .
$$

Likewise, for any $x \in \Omega$, there exists $x_{*}=x_{*}(x) \in \bar{\Omega}$ such that

$$
w_{\epsilon}(x)=w\left(x_{*}\right)+\frac{1}{\epsilon}\left|x_{*}-x\right|^{2} \text { and }\left|x_{*}-x\right|^{2} \leq \epsilon\left(w(x)-\min _{\bar{\Omega}} w\right) .
$$

(iv) If it holds for some non-empty open subset $\omega$ of $\Omega$ that $\inf _{\omega} v>-\infty$ and $\sup _{\omega} w<+\infty$, then

$$
\left|\nabla v^{\epsilon}\right| \leq \frac{2}{\epsilon^{\frac{1}{2}}}\left[\max _{\bar{\Omega}} v-\inf _{\omega} v\right]^{\frac{1}{2}} \text { and }\left|\nabla w_{\epsilon}\right| \leq \frac{2}{\epsilon^{\frac{1}{2}}}\left[\sup _{\omega} w-\min _{\bar{\Omega}} w\right]^{\frac{1}{2}}
$$

almost everywhere in $\omega$.

(v) The bounds for $\left|x^{*}-x\right|$ and $\left|x_{*}-x\right|$ in (iii) can be improved when $v$ and $w$ are more regular. In fact, if $|v(x)-v(y)| \leq m(|x-y|)$ for all $x, y \in \bar{\Omega}$ and for some non-negative continuous non-decreasing function $m:[0, \infty) \rightarrow[0, \infty)$ satisfying $m(0)=0$, then

$$
\left|x^{*}-x\right| \leq\left[\epsilon m\left(\left(2 \epsilon \sup _{\bar{\Omega}}|v|\right)^{1 / 2}\right)\right]^{1 / 2} .
$$

Analogously, if $|v(x)-v(y)| \leq m(|x-y|)$ for all $x, y \in \bar{\Omega}$, then

$$
\left|x_{*}-x\right| \leq\left[\epsilon m\left(\left(2 \epsilon \sup _{\bar{\Omega}}|w|\right)^{1 / 2}\right)\right]^{1 / 2} .
$$

Nevertheless, the bounds for $\left|x^{*}-x\right|$ and $\left|x_{*}-x\right|$ in (iiii) are generally sharp for semi-continuous functions.

Properties (ii)-(iii) can be found in [6]. To see Property (iv), we let $x_{0} \in \omega$ be a point of differentiablity of $v^{\epsilon}$, and estimate, for $x_{1} \in \Omega$,

$$
\begin{aligned}
v^{\epsilon}\left(x_{0}\right) & \geq v\left(\left(x_{1}\right)^{*}\right)-\frac{1}{\epsilon}\left|\left(x_{1}\right)^{*}-x_{0}\right|^{2} \\
& \geq v\left(\left(x_{1}\right)^{*}\right)-\frac{1}{\epsilon}\left|\left(x_{1}\right)^{*}-x_{1}\right|^{2}-\frac{2}{\epsilon}\left|\left(x_{1}\right)^{*}-x_{1}\right|\left|x_{1}-x_{0}\right|-\frac{1}{\epsilon}\left|x_{1}-x_{0}\right|^{2} \\
& =v^{\epsilon}\left(x_{1}\right)-\frac{2}{\epsilon}\left|\left(x_{1}\right)^{*}-x_{1}\right|\left|x_{1}-x_{0}\right|-\frac{1}{\epsilon}\left|x_{1}-x_{0}\right|^{2}, \text { for all } x_{0}, x_{1} \in \Omega,
\end{aligned}
$$


which implies, in view of Property (iii), that

$$
\frac{v^{\epsilon}\left(x_{1}\right)-v^{\epsilon}\left(x_{0}\right)}{\left|x_{1}-x_{0}\right|} \leq \frac{2}{\epsilon^{\frac{1}{2}}}\left|\max _{\bar{\Omega}} v-v\left(x_{1}\right)\right|+\frac{1}{\epsilon}\left|x_{1}-x_{0}\right| .
$$

Sending $x_{1} \rightarrow x_{0}$ and recalling Property (iii), we obtain the assertion. Property (国) follows from (iii) and the estimate

$$
\frac{1}{\epsilon}\left|x^{*}-x\right|^{2}=v\left(x^{*}\right)-v^{\epsilon}(x) \leq v\left(x^{*}\right)-v(x) \leq m\left(\left|x^{*}-x\right|\right) .
$$

The sharpness of the estimates for $\left|x^{*}-x\right|$ and $\left|x_{*}-x\right|$ in (iii) is demonstrated by the following example. Consider $\Omega=(-1,1)$. For $x \in[-1,1]$, define

$$
w(x)= \begin{cases}1 & \text { if } 2^{-(k+2)}<|x| \leq 2^{-(k+1)} \text { for some } k \geq 0 \\ 2-2^{k+1}|x| & \text { if } 2^{-(k+1)}<|x| \leq 2^{-k} \text { for some } k \geq 0 \\ 0 & \text { if } x=0\end{cases}
$$

Then $w \in L S C([-1,1]) \cap L^{\infty}(-1,1)$. For $k>1$, let $\epsilon_{k}=2^{-2(2 k+1)}$ and $x_{k}=2^{-(2 k+3)}$. We have

$$
w_{\epsilon_{k}}\left(x_{k}\right) \leq w(0)+\frac{1}{\epsilon_{k}}\left|x_{k}\right|^{2}=\frac{1}{16} .
$$

On the other hand, for $\left|y-x_{k}\right|<2^{-(2 k+4)}=\frac{1}{8} \sqrt{\epsilon_{k}}$, we have $w(y)>\frac{1}{2}$ and

$$
w(y)+\frac{1}{\epsilon_{k}}\left|y-x_{k}\right|^{2} \geq \frac{1}{2} .
$$

It follows that $\left|\left(x_{k}\right)_{*}-x_{k}\right| \geq \frac{1}{8} \sqrt{\epsilon_{k}}$.

We conclude the section with a simple lemma about the stability of envelops with respect to semi-continuity.

Lemma 2.1. Assume that $v \in U S C(\bar{\Omega})$ and $\inf _{\bar{\Omega}} v>-\infty$. Then for all sequences $\epsilon_{j} \rightarrow 0$ and $x_{j} \rightarrow x \in \Omega$, there holds

$$
\limsup _{j \rightarrow \infty} v^{\epsilon_{j}}\left(x_{j}\right) \leq v(x)
$$

Likewise, if $w \in L S C(\bar{\Omega})$ and $\sup _{\bar{\Omega}} w<+\infty$, then

$$
\liminf _{j \rightarrow \infty} w_{\epsilon_{j}}\left(x_{j}\right) \geq w(x) .
$$


Proof. We will only show the first assertion. Assume by contradiction that there exist some sequences $\epsilon_{j} \rightarrow 0, x_{j} \rightarrow x \in \Omega$ such that

$$
v^{\epsilon_{j}}\left(x_{j}\right) \geq v(x)+2 \delta \text { for some } \delta>0 .
$$

By the semi-continuity of $v$, there exists $\theta>0$ such that

$$
v(y) \leq v(x)+\delta \text { for all }|y-x|<\theta .
$$

By property (iii), there exists $\hat{x}_{j}$ such that

$$
v^{\epsilon_{j}}\left(x_{j}\right)=v\left(\hat{x}_{j}\right)-\frac{1}{\epsilon_{j}}\left|x_{j}-\hat{x}_{j}\right|^{2} \text { and }\left|x_{j}-\hat{x}_{j}\right|^{2} \leq \epsilon_{j}\left(\sup _{\bar{\Omega}} v-v\left(x_{j}\right)\right) \rightarrow 0,
$$

where we have used $\inf _{\bar{\Omega}} v>-\infty$. It then follows that $\left|\hat{x}_{j}-x\right|<\theta$ for all sufficiently large $j$ and so

$$
v^{\epsilon_{j}}\left(x_{j}\right) \leq v\left(\hat{x}_{j}\right) \leq v(x)+\delta,
$$

which amounts to a contradiction.

\section{The principle of propagation of touching points}

In this section, we prove Theorem 1.7. We will establish the propagation principle for more general operators of the form

$$
F[\psi]=\nabla^{2} \psi+L(\cdot, \psi, \nabla \psi)
$$

where $L: \Omega \times \mathbb{R} \times \mathbb{R}^{n} \rightarrow \mathcal{S}^{n \times n}$, under some structural assumptions on $L$ and $U$ which we will detail below. (Clearly, Definition 1.3 extends to this general setting.)

It is natural to require that $L$ be locally Lipschitz continuous. When $L$ is only Hölder continuous, the propagation principle fails in a manner similar to the nonuniqueness of first order ODE with non-Lipschitz right hand side. The following example is well-known: Consider the equation $\Delta \psi=|\nabla \psi|^{\gamma}$ with $\gamma \in(0,1)$, i.e. $F[\psi]=\nabla^{2} \psi-|\nabla \psi|^{\gamma} I$ and $U=\left\{M \in \mathcal{S}^{n \times n}: \operatorname{tr}(M)>0\right\}$. This equation admits $\psi(x) \equiv 0$ and $\hat{\psi}(x)=\frac{1}{(\lambda+1)(\lambda+n-1)^{\lambda}}|x|^{\lambda+1}$ as classical solutions, where $\lambda=\frac{1}{1-\gamma}$. As $\hat{\psi} \geq \psi$ on $\mathbb{R}^{n}$ and equality holds only at $x=0$, the propagation principle fails.

We note that the degenerate ellipticity condition (8) and local Lipschitz regularity of $L$ are far from enough to ensure the correctness of the propagation principle (even for rotationally symmetric and proper operators); see subsection 3.5 for counterexamples. 
The following structural conditions on $(F, U)$ are directly motivated by the conformal operator $A[\psi]$. First, we assume that $U$ satisfies

$$
A \in U, c \in(0,1) \Rightarrow c A \in U .
$$

Second, we assume that, for every $R>0$ and $\Lambda>0$, there exist $m \geq 0, \bar{\theta}>0$ and $C>0$ such that, for $x \in \Omega$ and $p \in \mathbb{R}^{n}$,

$$
\begin{aligned}
& \left|\nabla_{x} L(x, s, p)\right| \leq C|p|^{m} \quad \forall|s| \leq R, \\
& 0 \leq L\left(x, s^{\prime}, p\right)-L(x, s, p) \leq C\left(s^{\prime}-s\right)|p|^{m} I \quad \forall-R \leq s \leq s^{\prime} \leq R, \\
& p \cdot \nabla_{p} L(x, s, p)-L(s, x, p) \\
& +\theta \Lambda\left|\nabla_{p} L(x, s, p)\right| I-\theta I \leq C p \otimes p-\frac{1}{C}|p|^{m} I \quad \forall \theta \in[0, \bar{\theta}],|s| \leq R .
\end{aligned}
$$

Note that, (25) and (26) should be understood as inequalities between real symmetric matrices: $M \leq N$ if and only if $N-M$ is non-negative definite. Also, (25) implies that $L$ is non-decreasing in $s$.

Example 3.1. For all $m \geq 2$ and $\alpha, \beta \in C_{\text {loc }}^{0,1}(\mathbb{R})$ such that $\beta(s)>\beta_{0}>0$ for some constant $\beta_{0}, \alpha$ is non-decreasing and $\beta$ is non-increasing, the operator

$$
F[\psi]=\nabla^{2} \psi+\alpha(\psi) \nabla \psi \otimes \nabla \psi-\beta(\psi)|\nabla \psi|^{m} I
$$

satisfies conditions (24) $-(26)$.

We now state our principle of propagation of touching points for operators of the form (22).

Theorem 3.2. Let $\Omega \subset \mathbb{R}^{n}(n \geq 2)$ be a non-empty bounded open set, $L: \Omega \times \mathbb{R} \times$ $\mathbb{R}^{n} \rightarrow \mathcal{S}^{n \times n}$ be locally Lipschitz continuous and satisfy (24), (25) and (26) for some $m>1, F$ be given by (22) and $U$ be a non-empty open subset of $\mathcal{S}^{n \times n}$ satisfying (8) and (23). If $w \in L S C(\bar{\Omega})$ and $v \in U S C(\bar{\Omega})$ are respectively a supersolution and a subsolution of (9) in $\Omega$, and if $w \geq v$ in $\Omega$ and $w>v$ on $\partial \Omega$, then $w>v$ in $\Omega$.

Interchanging the role of $\psi$ and $-\psi$ and of $U$ and $\mathcal{S}^{n \times n} \backslash(-\bar{U})$ (where $-\bar{U}=$ $\{-M: M \in \bar{U}\}$ ), we see that an analogous result holds if one replaces (23) by

$$
A \in U, c \in(1, \infty) \Rightarrow c A \in U
$$


and (26) by: for every $R>0$ and $\Lambda>0$, there exist positive constants $\bar{\theta}, C>0$ such that, for $0<\theta \leq \bar{\theta}, x \in \Omega,|s| \leq R$ and $p \in \mathbb{R}^{n}$,

$$
\begin{aligned}
& p \cdot \nabla_{p} L(x, s, p)-L(x, s, p) \\
& \quad-\theta \Lambda\left|\nabla_{p} L(x, s, p)\right| I+\theta I \geq-C p \otimes p+\frac{1}{C}|p|^{m} I .
\end{aligned}
$$

We then obtain an equivalent statement of Theorem 3.2.

Theorem 3.3. Let $\Omega \subset \mathbb{R}^{n}(n \geq 2)$ be a non-empty bounded open set, $L: \Omega \times \mathbb{R} \times$ $\mathbb{R}^{n} \rightarrow \mathcal{S}^{n \times n}$ be locally Lipschitz continuous and satisfy (24), (25) and (28) for some $m>1, F$ be given by (22) and $U$ be a non-empty open subset of $\mathcal{S}^{n \times n}$ satisfying (8) and (27). If $w \in L S C(\bar{\Omega})$ and $v \in U S C(\bar{\Omega})$ are respectively a supersolution and $a$ subsolution of (9) in $\Omega$ and if $w \geq v$ in $\Omega$ and $w>v$ on $\partial \Omega$, then $w>v$ in $\Omega$.

Assuming the correctness of the above theorem for the moment, we proceed with the

Proof of Theorem 1.7. If $\beta>\beta_{0}>0$, the result is covered by Theorem 3.2. If $\beta<-\beta_{0}<0$, the result is covered by Theorem 3.3. It remains to consider the case $\beta \equiv 0$ and $\alpha$ is constant. The operator $F$ then takes the form

$$
F[\psi]=\nabla^{2} \psi+\alpha \nabla \psi \otimes \nabla \psi
$$

When $\alpha \neq 0$, we note that the functions $\tilde{w}=\frac{\alpha}{|\alpha|} e^{\alpha w}$ and $\tilde{v}=\frac{\alpha}{|\alpha|} e^{\alpha v}$ satisfy $\tilde{w} \in$ $L S C(\bar{\Omega}), \tilde{v} \in U S C(\bar{\Omega})$ and, in view of (10),

$$
\nabla^{2} \tilde{w}=|\alpha||\tilde{w}| F[w] \in \mathcal{S}^{n \times n} \backslash U \text { and } \nabla^{2} \tilde{v}=|\alpha||\tilde{v}| F[v] \in \bar{U} .
$$

Therefore, we can assume without loss of generality that $\alpha=0$, i.e.

$$
F[\psi]=\nabla^{2} \psi
$$

In this case, note that

$$
F\left[\psi+\mu|x|^{2}\right]=F[\psi]+2 \mu I
$$

An easy adaption of the proof of Theorem 3.2 below (but using (29) instead of Lemma 3.5) yields the result.

We turn now to the proof of Theorem 3.2 . 


\subsection{Error in regularizations}

The following result is a direct adaption of [6, Theorem 5.1] which estimates the error to (9) when making regularizations by lower and upper envelops.

Proposition 3.4. Assume $n \geq 2$. Let $\Omega \subset \mathbb{R}^{n}$ be a bounded open set, $U$ be an open subset of $\mathcal{S}^{n \times n}$ satisfying (8) $, L: \Omega \times \mathbb{R} \times \mathbb{R}^{n} \rightarrow \mathcal{S}^{n \times n}$ be a locally Lipschitz continuous function satisfying (24) and the second inequality in (25) for some $m \geq 0$, and $F$ be given by (22). For any $M>0$, there exists $a>0$ such that if $w \in L S C(\Omega)$ is a supersolution of (9) in $\Omega$ and if $w_{\epsilon}$ is punctually second order differentiable at a point $x \in \Omega$ and $\left|w_{\epsilon}(x)\right|+\left|w\left(x_{*}\right)\right| \leq M$, then

$$
F\left[w_{\epsilon}\right](x)-a\left|x_{*}-x\right|\left(1+\frac{1}{\epsilon}\left|x_{*}-x\right|\right)\left|\nabla w_{\epsilon}(x)\right|^{m} I \in \mathcal{S}^{n \times n} \backslash U .
$$

Analogously, if $v \in U S C(\Omega)$ is a subsolution of (9) in $\Omega$, and if $v^{\epsilon}$ is punctually second order differentiable at a point $x \in \Omega$ and $\left|v^{\epsilon}(x)\right|+\left|v\left(x^{*}\right)\right| \leq M$, then

$$
F\left[v^{\epsilon}\right](x)+a\left|x^{*}-x\right|\left(1+\frac{1}{\epsilon}\left|x^{*}-x\right|\right)\left|\nabla v_{\epsilon}(x)\right|^{m} I \in U .
$$

Proof. We only give the proof of the first assertion. The second assertion can be proved in a similar way.

We have

$$
w_{\epsilon}(x+z) \geq w_{\epsilon}(x)+\nabla w_{\epsilon}(x) \cdot z+\frac{1}{2} z^{T} \nabla^{2} w_{\epsilon}(x) z+o\left(|z|^{2}\right), \quad \text { as } z \rightarrow 0 .
$$

By the definition of $w_{\epsilon}$, we have

$$
w_{\epsilon}(x+z) \leq w\left(x_{*}+z\right)+\frac{1}{\epsilon}\left|x_{*}-x\right|^{2},
$$

and therefore, in view of (30),

$$
\begin{aligned}
w\left(x_{*}+z\right) & \geq w_{\epsilon}(x+z)-\frac{1}{\epsilon}\left|x_{*}-x\right|^{2} \\
& \geq P_{\epsilon}\left(x_{*}+z\right)+o\left(|z|^{2}\right), \quad \text { as } z \rightarrow 0,
\end{aligned}
$$

where $P_{\epsilon}$ is a quadratic polynomial with

$$
\begin{aligned}
P_{\epsilon}\left(x_{*}\right) & =w_{\epsilon}(x)-\frac{1}{\epsilon}\left|x_{*}-x\right|^{2}=w\left(x_{*}\right), \\
\nabla P_{\epsilon}\left(x_{*}\right) & =\nabla w_{\epsilon}(x), \\
\nabla^{2} P_{\epsilon}\left(x_{*}\right) & =\nabla^{2} w_{\epsilon}(x) .
\end{aligned}
$$


Since $w$ is a supersolution of (9), we thus have

$$
\nabla^{2} w_{\epsilon}(x)+L\left(x_{*}, w\left(x_{*}\right), \nabla w_{\epsilon}(x)\right)=F\left[P_{\epsilon}\right]\left(x_{*}\right) \in \mathcal{S}^{n \times n} \backslash U .
$$

On the other hand, in view of (24), (25) and $w\left(x_{*}\right)=w_{\epsilon}(x)-\frac{1}{\epsilon}\left|x_{*}-x\right|^{2} \leq w_{\epsilon}(x)$,

$L\left(x, w_{\epsilon}(x), \nabla w_{\epsilon}(x)\right)-L\left(x_{*}, w\left(x_{*}\right), \nabla w_{\epsilon}(x)\right) \leq C\left(\left|x-x_{*}\right|+\frac{1}{\epsilon}\left|x-x_{*}\right|^{2}\right)\left|\nabla w_{\epsilon}(x)\right|^{m} I$.

The conclusion is readily seen thanks to (8).

\subsection{First variation of $F[\psi]$}

As mentioned in the introduction, we would like to perturb a given function $\psi$ to another function $\tilde{\psi}$ in such a way that $F[\tilde{\psi}]$ is bounded from above/below by a multiple of $F[\psi]$ and with a favorable excess term. This will be important in controlling error accrued in other parts of the proof of Theorem 3.2 (e.g. in regularizations).

Lemma 3.5. Let $\Omega$ be an open bounded subset of $\mathbb{R}^{n}, n \geq 2, L: \Omega \times \mathbb{R} \times \mathbb{R}^{n} \rightarrow \mathcal{S}^{n \times n}$ be a locally Lipschitz continuous function satisfying (25) and (26) for some $m>1$, $F$ be given by (22), and $\psi: \Omega \rightarrow \mathbb{R} \cup\{ \pm \infty\}$. For any $M>0$, there exist positive constants $\mu_{0}, \alpha, \beta, \delta, K_{0}>0$, depending only on an upper bound of $M, L$ and $\Omega$, such that

$$
\mu_{0} \beta \sup _{\Omega} e^{-\beta \psi} \leq \frac{1}{2}
$$

and, for any $0<\mu<\mu_{0}, \tau \in \mathbb{R}$, the function $\tilde{\psi}_{\mu, \tau}=\psi+\mu\left(e^{\alpha|x|^{2}}+e^{-\beta \psi}-\tau\right)$ satisfies

$$
F\left[\tilde{\psi}_{\mu}\right] \geq\left(1-\mu \beta e^{-\beta \psi}\right) F[\psi]+\mu K_{0}\left[\left(1+|\nabla \psi|^{m}\right) I+\nabla \psi \otimes \nabla \psi\right]
$$

in the set

$$
\begin{gathered}
\Omega^{M, \delta}:=\{x \in \Omega: \psi \text { is punctually second order differentiable at } x \\
\left.|\psi(x)| \leq M, \text { and } e^{\alpha|x|^{2}}+e^{-\beta \psi(x)}-\tau \geq-\delta\right\}
\end{gathered}
$$

Proof. In the proof, $C$ will denote some large positive constant which may become larger as one moves from lines to lines but depends only on an upper bound for $M$, $L$ and $\Omega$. Eventually, we will choose large $\beta=\beta(C)>0$, small $\alpha=\alpha(\beta, M, C)>0$, and finally small $\mu_{0}=\mu_{0}(\alpha, \beta, M, C)>0$.

We set $\varphi(x)=e^{\alpha|x|^{2}}, f(\psi)=-e^{-\beta \psi}$ and abbreviate $\tilde{\psi}_{\mu}=\tilde{\psi}_{\mu, \tau}=\psi+\mu(\varphi-$ $f(\psi)-\tau)$. Note that $f^{\prime}(\psi)>0$. 
We assume in the sequel that $\alpha<1, \delta<1$ and

$$
\mu_{0} \sup _{\Omega}\left[1+f^{\prime}(\psi)\right] \leq \frac{1}{C}<\frac{1}{2}
$$

The following computation is done at a point in $\Omega^{M, \delta}$. We have

$$
\begin{aligned}
F\left[\tilde{\psi}_{\mu}\right] \geq(1-\mu & \left.f^{\prime}(\psi)\right) F[\psi]-\mu f^{\prime \prime}(\psi) \nabla \psi \otimes \nabla \psi+2 \mu \alpha \varphi I \\
& +L\left(x, \tilde{\psi}_{\mu}, \nabla \tilde{\psi}_{\mu}\right)-\left(1-\mu f^{\prime}(\psi)\right) L(x, \psi, \nabla \psi) .
\end{aligned}
$$

Noting that $\varphi-f(\psi)-\tau \geq-\delta$ in $\Omega^{M, \delta}$, we deduce from (25) and (32) that

$$
L\left(x, \tilde{\psi}_{\mu}, \nabla \tilde{\psi}_{\mu}\right) \geq L\left(x, \psi, \nabla \tilde{\psi}_{\mu}\right)-C \mu \delta\left(|\nabla \psi|^{m}+\mu^{m} \alpha^{m} \varphi^{m}\right) I .
$$

Therefore,

$$
\begin{aligned}
F\left[\tilde{\psi}_{\mu}\right] \geq(1-\mu & \left.f^{\prime}(\psi)\right) F[\psi]-\mu f^{\prime \prime}(\psi) \nabla \psi \otimes \nabla \psi \\
& +2 \mu \alpha\left(1-C \delta \mu^{m} \alpha^{m-1} \varphi^{m-1}\right) \varphi I-C \mu \delta|\nabla \psi|^{m} I \\
& +L\left(x, \psi, \nabla \tilde{\psi}_{\mu}\right)-\left(1-\mu f^{\prime}(\psi)\right) L(x, \psi, \nabla \psi) .
\end{aligned}
$$

We proceed to estimate $L\left(x, \psi, \nabla \tilde{\psi}_{\mu}\right)-\left(1-\mu f^{\prime}(\psi)\right) L(x, \psi, \nabla \psi)$. For $0 \leq t \leq \mu$, let

$$
g(t)=\frac{L\left(x, \psi, \nabla \tilde{\psi}_{t}\right)}{1-t f^{\prime}(\psi)}
$$

We have

$$
\begin{gathered}
\frac{d}{d t} g(t) \geq \frac{f^{\prime}(\psi)}{\left(1-t f^{\prime}(\psi)\right)^{2}}\left[L\left(x, \psi, \nabla \tilde{\psi}_{t}\right)-\nabla \tilde{\psi}_{t} \cdot \nabla_{p} L\left(x, \psi, \nabla \tilde{\psi}_{t}\right)\right. \\
\left.-\frac{C \alpha \varphi}{f^{\prime}(\psi)}\left|\nabla_{p} L\left(x, \psi, \nabla \tilde{\psi}_{t}\right)\right| I\right] .
\end{gathered}
$$

Thus, in view of (26) and (32), if $\alpha, \beta$ and $\delta$ satisfy

$$
\alpha \sup _{\Omega} \varphi\left[\frac{1}{f^{\prime}(\psi)}+1\right] \leq \frac{1}{C}
$$

then, with $\Lambda=8 C$ and $\theta=\frac{\alpha \varphi}{8 f^{\prime}(\psi)}$ in (26),

$$
\begin{aligned}
\frac{d}{d t} g(t) & \geq f^{\prime}(\psi)\left[-C \nabla \tilde{\psi}_{t} \otimes \nabla \tilde{\psi}_{t}+\frac{1}{C}\left|\nabla \tilde{\psi}_{t}\right|^{m} I\right]-\frac{1}{2} \alpha \varphi I \\
& \geq f^{\prime}(\psi)\left[-C \nabla \psi \otimes \nabla \psi+\frac{1}{C}|\nabla \psi|^{m} I\right]-\alpha \varphi I .
\end{aligned}
$$


This implies

$$
\begin{aligned}
& L\left(x, \psi, \nabla \tilde{\psi}_{\mu}\right)-\left(1-\mu f^{\prime}(\psi)\right) L(x, \psi, \nabla \psi) \\
& \quad=\left(1-\mu f^{\prime}(\psi)\right)[g(\mu)-g(0)] \\
& \quad \geq \mu f^{\prime}(\psi)\left[-C \nabla \psi \otimes \nabla \psi+\frac{1}{C}|\nabla \psi|^{m} I\right]-\mu \alpha \varphi I .
\end{aligned}
$$

Combining (33) and (35) and using (34), we obtain

$$
\begin{gathered}
F\left[\tilde{\psi}_{\mu}\right] \geq\left(1-\mu f^{\prime}(\psi)\right) F[\psi]+\mu \alpha \varphi I+\frac{1}{C} \mu\left(f^{\prime}(\psi)-C \delta\right)|\nabla \psi|^{m} I \\
+\mu\left[-f^{\prime \prime}(\psi)-C f^{\prime}(\psi)\right] \nabla \psi \otimes \nabla \psi
\end{gathered}
$$

We now fix $C$ and proceed with the choice of $\alpha, \beta, \delta$ and $\mu_{0}$. First, choosing $\beta \geq 2 C$ and recalling the definition of $f$, we have

$$
-f^{\prime \prime}(\psi)-C f^{\prime}(\psi)=\beta(\beta-C) e^{-\beta \psi} \geq \frac{1}{2} \beta f^{\prime}(\psi)
$$

Next, choose $\alpha$ such that (34) is satisfied and choose $\delta$ such that $f^{\prime}(\psi)-C \delta \geq \frac{1}{2} f^{\prime}(\psi)$. Finally, choose $\mu_{0}$ such that (32) holds. We hence obtain from (36) that

$$
F[\tilde{\psi}] \geq\left(1-\mu f^{\prime}(\psi)\right) F[\psi]+\mu \alpha \varphi I+\frac{1}{C} \mu f^{\prime}(\psi)|\nabla \psi|^{m} I+\frac{1}{2} \beta \mu f^{\prime}(\psi) \nabla \psi \otimes \nabla \psi .
$$

This completes the proof.

Lemma 3.6. Let $\Omega$ be an open bounded subset of $\mathbb{R}^{n}, n \geq 2, L: \Omega \times \mathbb{R} \times \mathbb{R}^{n} \rightarrow$ $\mathcal{S}^{n \times n}$ be a locally Lipschitz continuous function satisfying (25) and (26) for some $m>1, F$ be given by (22), and $\psi: \Omega \rightarrow \mathbb{R} \cup\{ \pm \infty\}$. There exist positive constants $\mu_{0}, \alpha, \beta, \delta, K_{0}>0$, depending only on an upper bound of $\sup _{\Omega}|\psi|, L$ and $\Omega$, such that, for any $0<\mu<\mu_{0}, \tau \in \mathbb{R}$, the function $\hat{\psi}_{\mu, \tau}=\psi-\mu\left(e^{\alpha|x|^{2}}+e^{-\beta \psi}-\tau\right)$ satisfies

$$
F\left[\hat{\psi}_{\mu}\right] \leq\left(1+\mu \beta e^{-\beta \psi}\right) F[\psi]-\mu K_{0}\left[\left(1+|\nabla \psi|^{m}\right) I+\nabla \psi \otimes \nabla \psi\right]
$$

in the set $\Omega^{M, \delta}$ defined by (31).

Proof. The proof is similar to that of Lemma 3.5 and is omitted. 


\subsection{Proof of Theorem 3.2}

Arguing by contradiction, we suppose that there exists $\gamma>0$ such that

$$
\max _{\bar{\Omega}}(v-w)=0 \quad \text { and } \quad(v-w)(x) \leq-\gamma, \quad \forall x \in \overline{\Omega \backslash \Omega_{\gamma}}
$$

where $\Omega_{\gamma}=\{x \in \Omega: \operatorname{dist}(x, \partial \Omega)>\gamma\}$.

For $\epsilon>0$, let $v^{\epsilon}$ and $w_{\epsilon}$ be the $\epsilon$-upper and $\epsilon$-lower envelops of $v$ and $w$ respectively (see Section 2). We note that

$$
v \leq v^{\epsilon} \leq \max _{\bar{\Omega}} v<+\infty \text { and } w \geq w_{\epsilon} \geq \min _{\bar{\Omega}} w>-\infty .
$$

In the sequel, we use $C$ to denote some positive constant which depends on $\max _{\bar{\Omega}} v, \min _{\bar{\Omega}} w, L$ and $\Omega$ but is always independent of $\epsilon$.

By Lemma 3.5, we can find $\bar{\mu}>0, \delta>0$ and a smooth positive function $f: \mathbb{R}^{n} \times \mathbb{R} \rightarrow(0, \infty)$, depending only on $\max _{\bar{\Omega}} v, \min _{\bar{\Omega}} w, L$ and $\Omega$, such that $f$ is decreasing with respect to the $s$-variable, $\bar{\mu} \sup _{\Omega}\left|\partial_{s} f\left(\cdot, v^{\epsilon}\right)\right| \leq \frac{1}{2}$ and, for $\mu \in(0, \bar{\mu})$, $\tau \in \mathbb{R}$ and $\tilde{v}_{\epsilon, \tau}=v^{\epsilon}+\mu\left(f\left(\cdot, v^{\epsilon}\right)-\tau\right)$, there holds

$$
F\left[\tilde{v}_{\epsilon, \tau}\right] \geq\left(1-\mu\left|\partial_{s} f\left(\cdot, v^{\epsilon}\right)\right|\right) F\left[v^{\epsilon}\right]+\frac{\mu}{C}\left(1+\left|\nabla v^{\epsilon}\right|^{m}\right) I
$$

in the set

$$
\begin{aligned}
& \tilde{\Omega}_{\epsilon}:=\left\{x \in \Omega_{\gamma / 2}: v^{\epsilon} \text { is punctually second order differentiable at } x,\right. \\
& \left.\qquad v^{\epsilon}(x) \geq \min _{\bar{\Omega}} w-1 \text { and } f\left(x, v^{\epsilon}(x)\right)-\tau \geq-\delta\right\} .
\end{aligned}
$$

Note that $\bar{\mu}$ and $\delta$ are independent of $\epsilon$. Furthermore, in view of (19), there exists $\bar{\eta}>0$ independent of $\epsilon$ such that, for all small $\epsilon$ and $\eta \in(0, \bar{\eta})$, one can (uniquely) find $\tau=\tau(\epsilon, \eta)$ such that the function $\xi_{\epsilon, \eta}:=\tilde{v}_{\epsilon, \tau}-w_{\epsilon}$ satisfies

$$
\max _{\bar{\Omega}} \xi_{\epsilon, \eta}=\eta \text { and } \xi_{\epsilon, \eta}<-\frac{\gamma}{2} \text { in } \overline{\Omega \backslash \Omega_{\gamma}} .
$$

Let $\Gamma_{\xi_{\epsilon, \eta}^{+}}$denote the concave envelope of $\xi_{\epsilon, \eta}^{+}:=\max \left\{\xi_{\epsilon, \eta}, 0\right\}$ on $\bar{\Omega}$. Then by (20), we have

$$
\nabla^{2} \xi_{\epsilon, \eta} \geq-\frac{4}{\epsilon} I \quad \text { a.e. in } \Omega_{\gamma}
$$

By [6, Lemma 3.5], we have

$$
\int_{\left\{\xi_{\epsilon, \eta}=\Gamma_{\xi_{\epsilon, \eta}^{+}}\right\}} \operatorname{det}\left(-\nabla^{2} \Gamma_{\xi_{\epsilon, \eta}^{+}}\right)>0
$$


which implies that the Lebesgue measure of $\left\{\xi_{\epsilon, \eta}=\Gamma_{\xi_{\epsilon, \eta}^{+}}\right\}$is positive. Then there exists $x_{\epsilon, \eta} \in\left\{\xi_{\epsilon, \eta}=\Gamma_{\xi_{\epsilon, \eta}^{+}}\right\} \cap \Omega_{\gamma}$ such that both of $v^{\epsilon}$ and $w_{\epsilon}$ are punctually second order differentiable at $x_{\epsilon, \eta}$,

$$
\begin{gathered}
0<\xi_{\epsilon, \eta}\left(x_{\epsilon, \eta}\right) \leq \eta \\
\left|\nabla \xi_{\epsilon, \eta}\left(x_{\epsilon, \eta}\right)\right|=\left|\nabla \tilde{v}_{\epsilon, \tau}\left(x_{\epsilon, \eta}\right)-\nabla w_{\epsilon}\left(x_{\epsilon, \eta}\right)\right| \leq C \eta
\end{gathered}
$$

and

$$
\nabla^{2} \xi_{\epsilon, \eta}\left(x_{\epsilon, \eta}\right)=\nabla^{2} \tilde{v}_{\epsilon, \tau}\left(x_{\epsilon, \eta}\right)-\nabla^{2} w_{\epsilon}\left(x_{\epsilon, \eta}\right) \leq 0 .
$$

From (38) and the definition of $\tilde{v}_{\epsilon, \tau}$, we have

$$
f\left(x_{\epsilon, \eta}, v^{\epsilon}\left(x_{\epsilon, \eta}\right)\right)-\tau>\frac{1}{\mu}\left(w_{\epsilon}\left(x_{\epsilon, \eta}\right)-v^{\epsilon}\left(x_{\epsilon, \eta}\right)\right) .
$$

Note that, as $w \geq v$ in $\Omega$, Lemma 2.1 implies that

$$
\liminf _{\epsilon \rightarrow 0, \eta \rightarrow 0}\left[w_{\epsilon}\left(x_{\epsilon, \eta}\right)-v^{\epsilon}\left(x_{\epsilon, \eta}\right)\right] \geq 0 .
$$

Hence, by shrinking $\mu$ and $\bar{\eta}$ if necessary, we may assume for all small $\epsilon$ that

$$
f\left(x_{\epsilon, \eta}, v^{\epsilon}\left(x_{\epsilon, \eta}\right)\right)-\tau \geq-\delta, \quad v^{\epsilon}\left(x_{\epsilon, \eta}\right) \geq \min _{\bar{\Omega}} w-1, \quad \text { and } w_{\epsilon}\left(x_{\epsilon, \eta}\right) \leq \max _{\bar{\Omega}} v+1
$$

We deduce that $x_{\epsilon, \eta} \in \tilde{\Omega}_{\epsilon, \delta}$ and thus obtain from (37) that

$$
F\left[\tilde{v}_{\epsilon, \tau}\right]\left(x_{\epsilon, \eta}\right) \geq\left(1-\mu\left|\partial_{s} f\left(x_{\epsilon, \eta}, v^{\epsilon}\left(x_{\epsilon, \eta}\right)\right)\right|\right) F\left[v^{\epsilon}\right]\left(x_{\epsilon, \eta}\right)+\frac{\mu}{C}\left(1+\left|\nabla v^{\epsilon}\left(x_{\epsilon, \eta}\right)\right|^{m}\right) I .
$$

Next, the proof of (21) implies that, for any unit vector $e$,

$$
\partial_{e} v^{\epsilon}\left(x_{\epsilon, \eta}\right) \geq-\frac{C}{\sqrt{\epsilon}} \text { and } \partial_{e} w_{\epsilon}\left(x_{\epsilon, \eta}\right) \leq \frac{C}{\sqrt{\epsilon}} .
$$

This together with (39) implies that, for all sufficiently small $\eta$,

$$
\left|\nabla \tilde{v}_{\epsilon, \tau}\left(x_{\epsilon, \eta}\right)\right|+\left|\nabla w_{\epsilon}\left(x_{\epsilon, \eta}\right)\right| \leq \frac{C}{\sqrt{\epsilon}} .
$$

Thus, by the local Lipschitz regularity of $L$,

$$
L\left(x_{\epsilon, \eta}, w_{\epsilon}\left(x_{\epsilon, \eta}\right), \nabla w_{\epsilon}\left(x_{\epsilon, \eta}\right)\right)-L\left(x_{\epsilon, \eta}, \tilde{v}_{\epsilon, \tau}\left(x_{\epsilon, \eta}\right), \nabla \tilde{v}_{\epsilon}\left(x_{\epsilon, \eta}\right)\right) \geq-C(\epsilon) \eta I .
$$

This together with (40) implies that

$$
F\left[w_{\epsilon}\right]\left(x_{\epsilon, \eta}\right) \geq F\left[\tilde{v}_{\epsilon, \tau}\right]\left(x_{\epsilon, \eta}\right)-C(\epsilon) \eta I .
$$


Recalling (42), we can find $\hat{\eta}=\hat{\eta}(\epsilon)$ such that, for $0<\eta<\hat{\eta}(\epsilon)$, there holds

$$
F\left[w_{\epsilon}\right]\left(x_{\epsilon, \eta}\right) \geq\left(1-\mu\left|\partial_{s} f\left(x_{\epsilon, \eta}, v^{\epsilon}\left(x_{\epsilon, \eta}\right)\right)\right|\right) F\left[v^{\epsilon}\right]\left(x_{\epsilon, \eta}\right)+\frac{\mu}{C}\left(1+\left|\nabla v^{\epsilon}\left(x_{\epsilon, \eta}\right)\right|^{m}\right) I .
$$

We next claim that

$$
\liminf _{\epsilon \rightarrow 0, \eta \rightarrow 0} \frac{1}{\epsilon}\left[\left|\left(x_{\epsilon, \eta}\right)_{*}-x_{\epsilon, \eta}\right|^{2}+\left|\left(x_{\epsilon, \eta}\right)^{*}-x_{\epsilon, \eta}\right|^{2}\right] \leq C \mu^{2} .
$$

Assuming this claim for now, we use Proposition 3.4 to find $a>0$ independent of $\epsilon$ and $\eta$ such that one has, in $\Omega_{\gamma}$,

$$
\begin{gathered}
F\left[w_{\epsilon}\right]\left(x_{\epsilon, \eta}\right)-a\left|\left(x_{\epsilon, \eta}\right)_{*}-x_{\epsilon, \eta}\right|\left(1+\frac{1}{\epsilon}\left|\left(x_{\epsilon, \eta}\right)_{*}-x_{\epsilon, \eta}\right|\right)\left|\nabla w_{\epsilon}\left(x_{\epsilon, \eta}\right)\right|^{m} I \in \mathcal{S}^{n \times n} \backslash U, \\
F\left[v^{\epsilon}\right]\left(x_{\epsilon, \eta}\right)+a\left|\left(x_{\epsilon, \eta}\right)^{*}-x_{\epsilon, \eta}\right|\left(1+\frac{1}{\epsilon}\left|\left(x_{\epsilon, \eta}\right)^{*}-x_{\epsilon, \eta}\right|\right)\left|\nabla v^{\epsilon}\left(x_{\epsilon, \eta}\right)\right|^{m} I \in \bar{U},
\end{gathered}
$$

where $x_{*}$ and $x^{*}$ are as in Section 2. The relations (44), (46) and (47) amount to a contradiction for sufficiently small $\mu$ thanks to (8) and (23). Therefore, to conclude the proof it suffices to prove the claim (45).

Pick some $\eta(\epsilon)<\hat{\eta}(\epsilon)$ such that $\eta(\epsilon) \rightarrow 0$ as $\epsilon \rightarrow 0$. Pick a sequence $\epsilon_{m} \rightarrow 0$ such that, for $x_{m}:=x_{\epsilon_{m}, \eta\left(\epsilon_{m}\right)}$, the sequence $\frac{1}{\epsilon_{m}}\left[\left|\left(x_{m}\right)^{*}-x_{m}\right|^{2}+\left|\left(x_{m}\right)_{*}-x_{m}\right|^{2}\right]$ converges to a limit which we will show to be no larger than $C \mu^{2}$. We will abbreviate $\tau_{m}:=$ $\tau\left(\epsilon_{m}, \eta\left(\epsilon_{m}\right)\right), v^{m}=v^{\epsilon_{m}}, w_{m}=w_{\epsilon_{m}}$. Without loss of generality, we may also assume that $x_{m} \rightarrow x_{0} \in \Omega, f\left(x_{m}, v^{m}\left(x_{m}\right)\right) \rightarrow f_{0}$ and $\tau_{m} \rightarrow \tau_{0}$.

As $\max _{\bar{\Omega}} \xi_{\epsilon, \eta}=\eta$, we have in view of (19) that

$$
\begin{aligned}
& v\left(x_{0}\right)-w\left(x_{0}\right)+\mu\left.f\left(x_{0}, v\left(x_{0}\right)\right)-\tau_{0}\right) \\
&=\lim _{m \rightarrow \infty}\left\{v^{m}\left(x_{0}\right)-w_{m}\left(x_{0}\right)+\mu\left(f\left(x_{0}, v^{m}\left(x_{0}\right)\right)-\tau_{m}\right)\right\} \leq 0 .
\end{aligned}
$$

On the other hand, by (38) and the fact that $f$ is decreasing in $s$, we have

$$
\begin{aligned}
f\left(x_{0}, \limsup _{m \rightarrow \infty} v^{m}\left(x_{m}\right)\right) & \leq f_{0}=\lim _{m \rightarrow \infty} f\left(x_{m}, v^{m}\left(x_{m}\right)\right) \\
& \leq \limsup _{m \rightarrow \infty} f\left(x_{m}, w_{m}\left(x_{m}\right)-\mu\left(f\left(x_{m}, v^{m}\left(x_{m}\right)\right)-\tau_{m}\right)\right) \\
& \leq f\left(x_{0}, \liminf _{m \rightarrow \infty} w_{m}\left(x_{m}\right)-\mu\left(f_{0}-\tau_{0}\right)\right)
\end{aligned}
$$

which implies, in view of Lemma 2.1 and the fact that $w \geq v$, that

$$
f\left(x_{0}, w\left(x_{0}\right)\right) \leq f\left(x_{0}, v\left(x_{0}\right)\right) \leq f_{0} \leq f\left(x_{0}, w\left(x_{0}\right)-\mu\left(f_{0}-\tau_{0}\right)\right),
$$


which further implies that

$$
0 \leq f_{0}-f\left(x_{0}, v\left(x_{0}\right)\right) \leq C \mu .
$$

Together with (48), this implies that

$$
v\left(x_{0}\right)-w\left(x_{0}\right)+\mu\left(f_{0}-\tau_{0}\right) \leq C \mu^{2} .
$$

We are now ready to wrap up the argument. As $\left(x_{\epsilon}\right)^{*}-x_{\epsilon} \rightarrow 0$ and $\left(x_{\epsilon}\right)_{*}-x_{\epsilon} \rightarrow 0$ as $\epsilon \rightarrow 0$, we have $\left(x_{m}\right)_{*} \rightarrow x_{0}$ and $\left(x_{m}\right)^{*} \rightarrow x_{0}$. As $v$ is upper semi-continuous and $w$ is lower semi-continuous, we have

$$
\limsup _{m \rightarrow \infty} v\left(\left(x_{m}\right)^{*}\right) \leq v\left(x_{0}\right) \text { and } \liminf _{m \rightarrow \infty} w\left(\left(x_{m}\right)_{*}\right) \geq w\left(x_{0}\right) .
$$

Thus, by the left half of (38),

$$
\begin{aligned}
0 & \leq \limsup _{m \rightarrow \infty} \frac{1}{\epsilon_{m}}\left[\left|\left(x_{\epsilon_{m}}\right)^{*}-x_{\epsilon_{m}}\right|^{2}+\left|\left(x_{\epsilon_{m}}\right)_{*}-x_{\epsilon_{m}}\right|^{2}\right] \\
& \leq \limsup _{m \rightarrow \infty}\left\{v\left(\left(x_{\epsilon_{m}}\right)^{*}\right)-w\left(\left(x_{\epsilon_{m}}\right)_{*}\right)+\mu\left(f\left(x_{\epsilon_{m}}, v^{\epsilon_{m}}\left(x_{\epsilon_{m}}\right)\right)-\tau\left(\epsilon_{m}, \eta\left(\epsilon_{m}\right)\right)\right]\right\} \\
& \leq v\left(x_{0}\right)-w\left(x_{0}\right)+\mu\left(f_{0}-\tau_{0}\right) \leq C \mu^{2} .
\end{aligned}
$$

We have proved (45), and thus concluded the proof.

\subsection{Proof of Corollary 1.8}

We will use a result from [7].

Theorem $3.7([7])$. Let $n \geq 1, \Omega \subset \mathbb{R}^{n}$ be a non-empty open set, and let $a, b \in$ $C^{0}\left(\Omega \times \mathbb{R} \times \mathbb{R}^{n}\right)$ satisfy

$$
a(x, z, p) \geq 0 \text { for all } x \in \Omega, z \in \mathbb{R}, p \in \mathbb{R}^{n},
$$

and $U \subset \mathcal{S}^{n \times n}$ be a non-empty open set satisfying (8). If $u \in L S C(\bar{\Omega})$ satisfies

$$
\Delta u \leq C \text { in } \Omega \text { in the viscosity sense, }
$$

and, for some subset $E$ of $\Omega$ of zero Lebesgue measure,

$$
a(x, u, D u) \nabla^{2} u+b(x, u, \nabla u) \in \mathcal{S}^{n \times n} \backslash U \text { in } \Omega \backslash E \text { in the viscosity sense, }
$$

then

$$
a(x, u, D u) \nabla^{2} u+b(x, u, \nabla u) \in \mathcal{S}^{n \times n} \backslash U \text { in } \Omega \text { in the viscosity sense. }
$$


Remark 3.8. This result was not stated as above in [7]. However, the proof of [7, Theorem 1.2] in effect yields the above result.

Proof of Corollary 1.8. Note that constant functions are solutions of (9) and the max of two subsolutions is a subsolution. It thus suffices to consider the case when

$$
\inf _{\bar{\Omega}} v>-\infty .
$$

By (14),

$$
\Delta v+(\alpha-n \beta)|\nabla v|^{2} \geq 0 \text { in } \Omega \backslash E \text { in the viscosity sense. }
$$

Note that when (16) holds, then the function $\tilde{u}=e^{-\frac{1}{|\alpha-n \beta|} v}$ satisfies

$$
\Delta \tilde{u} \leq 0 \text { in } \Omega \backslash E \text { in the viscosity sense. }
$$

As $\tilde{u}>0$ in $\Omega \backslash E$ and $E$ has zero capacity, the maximum principle then implies that $\tilde{u}>\frac{1}{c}>0$ in $\bar{\Omega} \backslash E$, and hence $\sup _{\bar{\Omega} \backslash E} v<+\infty$. Thus we can assume without loss of generality that (15) holds.

In view of the comparison principle Theorem 1.7(b), it suffices to show that

$$
F[v] \in \bar{U} \text { in } \Omega \text { in the viscosity sense, }
$$

where we define, for $x \in E$,

$$
v(x)=\limsup _{y \rightarrow x, y \in \Omega \backslash E} v(y)<+\infty .
$$

Indeed, we note that, for $C>|\alpha-n \beta|$, the function $u=-e^{C v} \in L S C(\bar{\Omega})$ satisfies $\inf _{\bar{\Omega}} u>-\infty, \sup _{\bar{\Omega}} u<0$ and

$$
\Delta u=C u\left(\Delta v+C|\nabla v|^{2}\right) \leq 0 \text { in } \Omega \backslash E \text { in the viscosity sense. }
$$

Since $E$ has zero capacity, it follows that

$$
\Delta u \leq 0 \text { in } \Omega \text { in the viscosity sense. }
$$

An application of Theorem 3.7 (to the set $\tilde{U}=\mathcal{S}^{n \times n} \backslash(-\bar{U})$ ) then implies that $F[v]=\frac{1}{C} \frac{\nabla^{2} u}{u}-\frac{1}{C} \frac{\nabla u \otimes \nabla u}{u^{2}}+L\left(x, \frac{1}{C} \ln (-u), \frac{\nabla u}{C u}\right) \in \bar{U}$ in $\Omega$ in the viscosity sense, which proves (49), and hence the assertion. 


\subsection{Counterexamples to the propagation principle}

It this section, we give examples to illustrate that (8), i.e. degenerate ellipticity, the properness and regularity of $L$ is insufficient to ensure the correctness of the propagation principle. These examples will also illustrate the importance of various technical assumptions in Theorems 1.7 and 3.2 .

Let $a, b \in C_{l o c}^{1}([0, \infty))$ and consider for now a rotationally invariant operator $F$ of the form

$$
F[\psi]=\nabla^{2} \psi+a(|\nabla \psi|) \nabla \psi \otimes \nabla \psi+b(|\nabla \psi|) I .
$$

In other words, we have

$$
L(p)=a(|p|) p \otimes p-b(|p|) I
$$

Note that although $a, b$ are locally differentiable, $L$ is in general only locally Lipschitz. $L$ is locally differentiable if and only if $b^{\prime}(0)=0$.

The following example suggests that some delicate attention should be paid if one allows $m=1$ in condition (26) (in the context of Theorem 3.2).

Proposition 3.9. Let $a, b \in C^{1}([0, \infty))$ and $F$ be of the form (50). If

$$
b(0)=0 \text { and } b^{\prime}(0) \neq 0,
$$

then the propagation principle does not hold for $F$, namely there exist a bounded domain $\Omega \in \mathbb{R}^{n}$, a non-empty open set $U \subset \mathcal{S}^{n \times n}$ satisfying (8), and a supersolution $w \in C^{2}(\bar{\Omega})$ and a subsolution $v \in C^{2}(\bar{\Omega})$ of (9) in $\Omega$ such that $w>v$ on $\partial \Omega$, but $\min _{\bar{\Omega}}(w-v)=0$.

Proof. Considering $F[-\psi]$ instead of $F[\psi]$ if necessary, we can assume without loss of generality that

$$
b^{\prime}(0)<0
$$

Let $U$ be the set of positive definite symmetric $n \times n$ matrices. Note that $v \equiv 0$ is a solution of (9) on $\mathbb{R}^{n}$. Since $L$ is independent of $\psi$, it suffices to exhibit a bounded domain $\Omega$, and a supersolution $w \in C^{2}(\bar{\Omega})$ of $(9)$ in $\Omega$ such that $w>0$ on $\partial \Omega$, but $\min _{\bar{\Omega}} w=0$.

In view of (51) and the fact that $b(0)=0$, there exists some $r_{0}>0$ and $\delta>0$ such that

$$
b(s)<0 \text { and } r_{0}>\frac{s}{|b(s)|} \text { for all } s \in(0, \delta) .
$$


Let $\Omega=\left\{r_{0}-1<|x|<r_{0}+1\right\}$ and $w(x)=w(|x|)$ for some $w \in C^{2}\left(\left[r_{0}-1, r_{0}+1\right]\right)$ satisfying $w\left(r_{0}\right)=w^{\prime}\left(r_{0}\right)=0$ and

$$
\begin{aligned}
& w^{\prime}(r) \in(-\delta, 0) \text { for } r \in\left[r_{0}-1, r_{0}\right), \\
& w^{\prime}(r) \in(0, \delta) \text { for } r \in\left(r_{0}, r_{0}+1\right] .
\end{aligned}
$$

Then $w>0$ on $\partial \Omega$ and $\min _{\bar{\Omega}} w=0$.

To conclude the proof, we check that $F[w]$ is not positive definite. Indeed, the eigenvalues of $F[w]$ are given by

$\lambda(F[w])=(\mu, \nu, \ldots, \nu)$ where $\mu=w^{\prime \prime}+a\left(\left|w^{\prime}\right|\right)\left|w^{\prime}\right|^{2}+b\left(\left|w^{\prime}\right|\right)$ and $\nu=\frac{1}{r} w^{\prime}+b\left(\left|w^{\prime}\right|\right)$.

Now, for $r<r_{0}$, we have $w^{\prime}<0$ (thanks to (53)) and $b\left(\left|w^{\prime}\right|\right)<0$ and so $\nu<0$. For $r>r_{0}$, we have, in view of (52) and (54),

$$
\nu=w^{\prime}\left(\frac{1}{r}-\frac{\left|b\left(w^{\prime}\right)\right|}{w^{\prime}}\right)<w^{\prime}\left(\frac{1}{r}-\frac{1}{r_{0}}\right)<0 .
$$

Also, $\nu=0$ when $r=r_{0}$. It thus follows that $\nu \leq 0$ in $\left(r_{0}-1, r_{0}+1\right)$, i.e. $F[w]$ is not positive definite. The proof is complete.

The previous result show that the propagation principle does not hold for general operators of the form (22). However, it is easy to see that the function $L$ in Proposition 3.9 is Lipschitz but not $C^{1}$. We will next construct some counterexamples with smooth $L$.

For $\alpha \in \mathbb{R}$, consider the rotationally invariant operator

$$
F[\psi]=\nabla^{2} \psi-\left(\psi^{3}|\nabla \psi|^{10}+\alpha \psi|\nabla \psi|^{6}+|\nabla \psi|^{4}\right) I,
$$

i.e.

$$
L(s, p)=-\left(s^{3}|p|^{10}+\alpha s|p|^{6}+|p|^{4}\right) I,
$$

which is an analytic function of $s$ and $p$. Note that neither condition (26) nor condition (28) is satisfied for this function $L$. Note also that the leading part of $L(s, p)$ changes sign as $s$ varies - this should be compared the assumption that $\beta(w)$ is of one sign in Theorem 1.7 .

Proposition 3.10. Let $n \geq 2, U$ be the set of positive definite symmetric $n \times n$ matrices, and $F$ be of the form (55) for some $\alpha<-\frac{5}{2}$. Then the propagation principle does not hold: there exists a bounded domain $\Omega$, a supersolution $w$ and a subsolution $v$ of (9) in $\Omega$ such that $w>v$ on $\partial \Omega$ but $\min _{\bar{\Omega}}(w-v)=0$. 
Proof. Fix some $r_{0}>0$. For $t \in \mathbb{R}$, let

$$
\psi_{t}(x)=\psi_{t}(r)=t^{\frac{1}{3}}\left|r-r_{0}\right|^{\frac{2}{3}}, \quad \text { where } r=|x| \text {. }
$$

The eigenvalues of $F\left[\psi_{t}\right]$ are $\left(\lambda_{1, t}, \lambda_{2, t}, \ldots, \lambda_{2, t}\right)$ where

$$
\begin{aligned}
\lambda_{1, t} & =\psi_{t}^{\prime \prime}-\psi_{t}^{3}\left|\psi_{t}^{\prime}\right|^{10}-\alpha \psi_{t}\left|\psi_{t}^{\prime}\right|^{6}-\left|\psi_{t}^{\prime}\right|^{4} \\
& =-\frac{2}{59049} \frac{t^{\frac{1}{3}}\left(8 P_{4}(t)+6561\right)}{\left|r-r_{0}\right|^{\frac{4}{3}}}, \\
\lambda_{2, t} & =\frac{1}{r} \psi_{t}^{\prime}-\psi_{t}^{3}\left|\psi_{t}^{\prime}\right|^{10}-\alpha \psi_{t}\left|\psi_{t}^{\prime}\right|^{6}-\left|\psi_{t}^{\prime}\right|^{4} \\
& =-\frac{2}{59049} \frac{t^{\frac{1}{3}}\left(8 P_{4}(t)-19683 \frac{r-r_{0}}{r}\right)}{\left|r-r_{0}\right|^{\frac{4}{3}}},
\end{aligned}
$$

and where $P_{4}(t)=64 t^{4}+324 \alpha t^{2}+729 t$.

Note that $P_{4}(0)=0$, and, as $\alpha<-\frac{5}{2}$,

$$
\begin{aligned}
& P_{4}(-2)=-434+1296 \alpha<-3674, \\
& P_{4}\left(\frac{9}{4}\right)=\frac{6561}{4}(2+\alpha)<-\frac{6561}{8} .
\end{aligned}
$$

It follows that the equation $8 P_{4}(t)+6561=0$ has four roots $t_{1}, \ldots, t_{4}$ satisfying $t_{1}<-2<t_{2}<0<t_{3}<\frac{9}{4}<t_{4}$. In particular, we have $\lambda_{1, t_{i}}(r)=0$ for $r \neq r_{0}$, $i=1, \ldots, 4$. Also, from the expression of $\lambda_{2, t}$, we can find some small $\delta>0$ such that

$$
t_{i} \lambda_{2, t_{i}}(r)>0 \text { for } r \neq r_{0},\left|r-r_{0}\right| \leq \delta .
$$

In addition, there exists $t_{0}<t_{1}$ such that

$$
\lambda_{1, t_{0}}(r)>0 \text { and } \lambda_{2, t_{0}}(r)>0 \text { for } r \neq r_{0},\left|r-r_{0}\right| \leq \delta .
$$

We define,

$$
\begin{aligned}
& w(x)=w(r)= \begin{cases}\psi_{t_{4}}(r) & \text { for } r_{0} \leq r \leq r_{0}+\delta, \\
\psi_{t_{2}}(r) & \text { for } r_{0}-\delta \leq r<r_{0},\end{cases} \\
& v(x)=v(r)= \begin{cases}\psi_{t_{3}}(r) & \text { for } r_{0} \leq r \leq r_{0}+\delta, \\
\psi_{t_{0}}(r) & \text { for } r_{0}-\delta \leq r<r_{0}\end{cases}
\end{aligned}
$$

It is readily seen that $w$ and $v$ are respectively a supersolution and a subsolution of (9) in $\Omega=\left\{\left|r-r_{0}\right|<\delta\right\}, w \geq v$ in $\Omega$ and $\{w=v\}=\left\{r=r_{0}\right\}$. This finishes the proof. 
The previous example can be modified to give a counterexample to the propagation principle with $L$ being non-increasing in $s$.

Proposition 3.11. Let $n \geq 2$ and $U$ be the set of positive definite symmetric $n \times n$ matrices. There exists a smooth function $L: \mathbb{R} \times \mathbb{R}^{n} \rightarrow \mathcal{S}^{n \times n}$ such that $L$ is nonincreasing in $s$ but the propagation principle does not hold for $F$ of the form (22): there exists a bounded domain $\Omega$, a supersolution $w$ and a subsolution $v$ of (9) in $\Omega$ such that $w>v$ on $\partial \Omega$ but $\min _{\bar{\Omega}}(w-v)=0$.

Proof. For $\alpha \in \mathbb{R}$ to be fixed, consider

$$
\tilde{L}(s, p)=-\left(s^{3}|p|^{10}+\alpha s|p|^{6}+\frac{1}{100}|p|^{4}\right) I .
$$

We first show that the propagation principle does not hold for $\tilde{F}=\nabla^{2}+\tilde{L}$ as in the proof of Proposition 3.10 .

Fix some $r_{0}>0$. For $t \in \mathbb{R}$, define $\psi_{t}$ by (56). The eigenvalues of $\tilde{F}\left[\psi_{t}\right]$ are $\left(\lambda_{1, t}, \lambda_{2, t}, \ldots, \lambda_{2, t}\right)$ where

$$
\begin{aligned}
\lambda_{1, t} & =\psi_{t}^{\prime \prime}-\psi_{t}^{3}\left|\psi_{t}^{\prime}\right|^{10}-\alpha \psi_{t}\left|\psi_{t}^{\prime}\right|^{6}-\frac{1}{100}\left|\psi_{t}^{\prime}\right|^{4} \\
& =-\frac{2}{1476225} \frac{t^{\frac{1}{3}}\left(2 \tilde{P}_{4}(t)+164025\right)}{\left|r-r_{0}\right|^{\frac{4}{3}}} \\
\lambda_{2, t} & =\frac{1}{r} \psi_{t}^{\prime}-\psi_{t}^{3}\left|\psi_{t}^{\prime}\right|^{10}-\alpha \psi_{t}\left|\psi_{t}^{\prime}\right|^{6}-\frac{1}{100}\left|\psi_{t}^{\prime}\right|^{4} \\
& =-\frac{2}{1476225} \frac{t^{\frac{1}{3}}\left(2 \tilde{P}_{4}(t)-492075 \frac{r-r_{0}}{r}\right)}{\left|r-r_{0}\right|^{\frac{4}{3}}}
\end{aligned}
$$

and where $\tilde{P}_{4}(t)=6400 t^{4}+32400 \alpha t^{2}+729 t$.

We next fix $\alpha=-\frac{36}{25}$. Then $\tilde{P}_{4}(-2)=-85682, \tilde{P}_{4}\left(-\frac{8}{5}\right)=-\frac{1966568}{25}, \tilde{P}_{4}\left(\frac{8}{5}\right)=$ $-\frac{1908248}{25}, \tilde{P}_{4}(2)=-82766$ and so the equation $2 \tilde{P}_{4}(t)+164025=0$ has four roots $\tilde{t}_{1}, \ldots, \tilde{t}_{4}$ satisfying $\tilde{t}_{1}<-2<\tilde{t}_{2}<-\frac{8}{5}<\frac{8}{5}<\tilde{t}_{3}<2<\tilde{t}_{4}$. Note that $\lambda_{1, \tilde{t}_{i}}(r)=0$ for $r \neq r_{0}, i=1, \ldots, 4$. Also, we can find some small $\delta>0$ such that

$$
\tilde{t}_{i} \lambda_{2, \tilde{t}_{i}}(r)>0 \text { for } r \neq r_{0},\left|r-r_{0}\right| \leq \delta, i \in\{2,3,4\} .
$$

As $\tilde{P}_{4}(-3)=96309>0$, we can also assume for $\tilde{t}_{0}=-3$ that

$$
\lambda_{1, \tilde{t}_{0}}(r)>0 \text { and } \lambda_{2, \tilde{t}_{0}}(r)>0 \text { for } r \neq r_{0},\left|r-r_{0}\right| \leq \delta
$$


We define,

$$
\begin{aligned}
& w(x)=w(r)= \begin{cases}\psi_{\tilde{t}_{4}}(r) & \text { for } r_{0} \leq r \leq r_{0}+\delta, \\
\psi_{\tilde{t}_{2}}(r) & \text { for } r_{0}-\delta \leq r<r_{0},\end{cases} \\
& v(x)=v(r)= \begin{cases}\psi_{\tilde{t}_{3}}(r) & \text { for } r_{0} \leq r \leq r_{0}+\delta, \\
\psi_{\tilde{t}_{0}}(r) & \text { for } r_{0}-\delta \leq r<r_{0} .\end{cases}
\end{aligned}
$$

It is readily seen that $w$ and $v$ are respectively a supersolution and a subsolution of (9) for the operator $\tilde{F}[\psi]=\nabla^{2} \psi+\tilde{L}(\psi, \nabla \psi)$ in $\Omega=\left\{\left|r-r_{0}\right|<\delta\right\}, w \geq v$ in $\Omega$ and $\{w=v\}=\left\{r=r_{0}\right\}$.

Now we proceed to modify $\tilde{L}$ to our desired $L$ as $\tilde{L}$ is not non-decreasing in $s$. We note that, as $\left|\tilde{t}_{2}\right|>\frac{8}{5}$ and $\left|\tilde{t}_{3}\right|>\frac{8}{5},(w(x), \nabla w(x))$ and $(v(x), \nabla v(x))$ belong to the set

$$
N:=\left\{(s, p) \in \mathbb{R} \times \mathbb{R}^{n}: s|p|^{2} \in \mathbb{R} \backslash\left(-\frac{32}{45}, \frac{32}{45}\right)\right\} \text { for all } x \in \Omega \backslash\left\{r=r_{0}\right\} .
$$

As $\partial_{s} \tilde{L}(s, p)=-|p|^{6}\left(3 s^{2}|p|^{4}+\alpha\right) I=-|p|^{6}\left(3 s^{2}|p|^{4}-\frac{36}{25}\right) I$, we see that $\tilde{L}$ is nonincreasing in $s$ for $(s, p) \in N$.

Next, note that, for a fixed $p \neq 0$,

$$
\tilde{L}\left(-\frac{32}{45}|p|^{-2}, p\right)=-\frac{245821}{364500}|p|^{4} I<0<\frac{238531}{364500}|p|^{4} I=\tilde{L}\left(\frac{32}{45}|p|^{-2}, p\right) .
$$

Therefore, there exists a smooth function $L: \mathbb{R} \times \mathbb{R}^{n} \rightarrow \mathcal{S}^{n \times n}$ which is non-increasing in $s$ such that $L \equiv \tilde{L}$ in $N$ (e.g. by smoothly interpolating in $s$ the values of $\tilde{L}$ on the boundary of $N)$. Then $w$ and $v$ are also a supersolution and a subsolution of (9) for the operator $F[\psi]=\nabla^{2} \psi+L(\psi, \nabla \psi)$ in $\Omega$. This completes the proof.

\section{Perron's method}

We begin with the

Proof of Theorem 1.4. The conclusion a direct consequence of Theorem 1.7(b).

In the rest of this section, we prove Theorem 1.5. We introduce some notations. For $O \subset \mathbb{R}^{n}, \xi: O \rightarrow[-\infty,+\infty]$, let

$$
\begin{gathered}
\xi^{*}(x):=\lim _{r \rightarrow 0^{+}} \sup \{\xi(y)|y \in O,| y-x \mid<r\}, \\
\xi_{*}(x):=\lim _{r \rightarrow 0^{+}} \inf \{\xi(y)|y \in O,| y-x \mid<r\} .
\end{gathered}
$$


It is easy to see that, if $\xi^{*}(x)<+\infty$ for all $x \in O$, then $\xi^{*} \in U S C(O)$. Likewise, if $\xi_{*}(x)>-\infty$ for all $x \in O$, then $\xi_{*} \in L S C(O)$.

$\xi^{*}$ is called the upper semicontinuous envelope of $\xi$, it is the smallest upper semicontinuous function satisfying $\xi \leq \xi^{*}$ in $O$. Similarly, $\xi_{*}$ is called the lower semicontinuous envelope of $\xi$, it is the largest lower semicontinuous function satisfying $\xi \geq \xi_{*}$ in $O$.

Note that, for any constant $c, F[c]=0 \in \partial U$. Thus, replacing $v$ by $\max (v, c)$ with some $c<\inf _{\partial \Omega} w$ and $w$ by $\min \left(w, c^{\prime}\right)$ with some $c^{\prime}>\sup _{\partial \Omega} v$ if necessary, we can assume that

$$
-\infty<\inf _{\bar{\Omega}} v \leq \sup _{\bar{\Omega}} w<+\infty .
$$

Here we have used the fact that the maximum of two subsolutions is a subsolution and the minimum of two supersolutions is a supersolution.

Note that by hypotheses, $w \geq v$ in $\Omega$. Define

$$
\begin{aligned}
u(x):=\quad \inf \{\xi(x) \mid & v \leq \xi \leq w \text { in } \bar{\Omega}, \xi=v=w \text { on } \partial \Omega \\
& \xi \in L S C(\bar{\Omega}), \xi \text { is a supersolution of (9) in } \Omega\} .
\end{aligned}
$$

Clearly

$$
\inf _{\bar{\Omega}} u \geq \inf _{\bar{\Omega}} v>-\infty
$$

We will prove that the above defined $u$ satisfies the requirement of Theorem 1.5.

Lemma 4.1. Let $O \subset \mathbb{R}^{n}$ be an open set, $L: O \times \mathbb{R} \times \mathbb{R}^{n} \rightarrow \mathcal{S}^{n \times n}$ be continuous, $F$ be given by (22), and let $\mathcal{F}$ be a family of supersolutions of (9) in $O$. Let

$$
\eta(x):=\inf \{\xi(x) \mid \xi \in \mathcal{F}\}, \quad x \in O
$$

Assume that $\eta_{*}(x)>-\infty \forall x \in O$. Then $\eta_{*}$ is a supersolution of (9) in $O$.

Proof. Suppose for some $x \in O$ that there exists a polynomial $P$ of the form

$$
P(y):=a+p \cdot(y-x)+\frac{1}{2}(y-x)^{t} M(y-x),
$$

with $a \in \mathbb{R}, p \in \mathbb{R}^{n}, M \in \mathcal{S}^{n \times n}$, such that, for some $\epsilon>0$,

$$
P(x)=\eta_{*}(x) \text { and } P(y) \leq \eta_{*}(y) \quad \forall|y-x|<\epsilon .
$$

We will show that

$$
F[P](x) \in \mathcal{S}^{n \times n} \backslash U
$$


It is standard that this implies that $\eta_{*}$ is a supersolution of (9) in the sense of Definition 1.3.

By the definition of $\eta_{*}$, there exists $r_{i} \rightarrow 0^{+},\left|x_{i}-x\right|<r_{i}$ such that

$$
\inf _{B_{r_{i}}(x)} \eta \leq \eta\left(x_{i}\right) \leq \inf _{B_{r_{i}}(x)} \eta+\frac{1}{i} \leq \eta_{*}(x)+\frac{1}{i} \text { and } \eta\left(x_{i}\right) \rightarrow \eta_{*}(x) .
$$

Moreover, there exists $\xi_{i} \in \mathcal{F}$, such that $\xi_{i} \geq \eta \geq \eta_{*}$ and

$$
0 \leq \xi_{i}\left(x_{i}\right)-\eta\left(x_{i}\right)<\frac{1}{i}
$$

We see from the above that

$$
\xi_{i} \geq \eta \geq \eta_{*} \geq P \quad \text { in } B_{\epsilon}(x),
$$

and

$$
\xi_{i}\left(x_{i}\right) \rightarrow \eta_{*}(x)=P(x) .
$$

For every $0<2 \delta<\min \{\epsilon, \operatorname{dist}(x, \partial O)\}$, consider

$$
P_{\delta}(y):=P(y)-\delta|y-x|^{2} .
$$

Then

$\xi_{i} \geq P_{\delta} \quad$ in $B_{\epsilon}(x), \quad \xi_{i} \geq P_{\delta}+\delta^{3} \quad$ in $B_{\epsilon}(x) \backslash B_{\delta}(x)$, and $\xi_{i}\left(x_{i}\right)-P_{\delta}\left(x_{i}\right) \rightarrow 0$.

It follows that there exists $\beta_{i}=\circ(1) \geq 0$ and $x_{i}^{*} \in B_{\delta}(x)$ such that

$$
\xi_{i}(y) \geq P_{\delta}(y)+\beta_{i}, \quad \text { in } B_{\epsilon}(x), \quad \xi_{i}\left(x_{i}^{*}\right)=P_{\delta}\left(x_{i}^{*}\right)+\beta_{i} .
$$

As $\xi_{i}$ is also a supersolution of (9) in $O$. Thus,

$$
F\left[P_{\delta}+\beta_{i}\right]\left(x_{i}^{*}\right) \in \mathcal{S}^{n \times n} \backslash U .
$$

Claim. $x_{i}^{*} \rightarrow x$.

Indeed, after passing to a subsequence, $x_{i}^{*} \rightarrow \bar{x}$, for some $\bar{x}$ satisfying $|\bar{x}-x| \leq \delta$. By (60) and the definition of $\eta$ and $\eta_{*}$,

$$
\eta_{*}\left(x_{i}^{*}\right)-\beta_{i} \leq \xi_{i}\left(x_{i}^{*}\right)-\beta_{i}=P_{\delta}\left(x_{i}^{*}\right) .
$$

Sending $i$ to infinity in the above, and using the lower-semicontinuity property of $\eta_{*}$, we have $\eta_{*}(\bar{x}) \leq P_{\delta}(\bar{x})=P(\bar{x})-\delta|\bar{x}-x|^{2}$. On the other hand, $P(\bar{x}) \leq \eta_{*}(\bar{x})$ according to (58). Thus $\bar{x}=x$, and the claim is proved.

With the convergence of $x_{i}^{*}$ to $x$ and of $\beta_{i}$ to 0 , sending $\delta$ to 0 and $i$ to $\infty$ in (61) yields (59). Lemma 4.1 is established. 
Proof of Theorem 1.5. We know that

$$
\max \left(v, u_{*}\right) \leq u \leq \min \left(u^{*}, w\right), \quad \text { in } \bar{\Omega},
$$

where $u$ is defined by (57). Clearly,

$$
v=u_{*}=u=u^{*}=w, \quad \text { on } \partial \Omega,
$$

By Lemma 4.1, $u_{*}$ is a supersolution of (9) in $\Omega$. By the comparison principle Theorem 1.7 (ii), $u_{*} \geq v$. Hence, by the definition of $u, u \leq u_{*}$ in $\bar{\Omega}$. Thus $u=u_{*}$ in $\bar{\Omega}$, and $u$ is a supersolution of (9) in $\Omega$.

Note that

$$
\sup _{\bar{\Omega}} u^{*} \leq \sup _{\bar{\Omega}} w<+\infty
$$

Claim. $u^{*}$ is a subsolution of (9) in $\Omega$.

To prove this claim, we follow Ishii's argument ([21]). Indeed, if the claim does not hold, there exist $x \in \Omega$ and some quadratic polynomial

$$
P(y)=a+p \cdot(y-x)+\frac{1}{2}(y-x)^{t} M(y-x),
$$

with $a \in \mathbb{R}, p \in \mathbb{R}^{n}, M \in \mathcal{S}^{n \times n}$, such that for some $\bar{\epsilon}>0$

$$
P(y) \geq u^{*}(y) \text { for } y \in B_{\bar{\epsilon}}(x), \quad P(x)=u^{*}(x),
$$

but

$$
F[P](x) \in \mathcal{S}^{n \times n} \backslash \bar{U} .
$$

Since $\mathcal{S}^{n \times n} \backslash \bar{U}$ is open, there exists $0<2 \bar{\delta}<\min \left\{\bar{\epsilon}^{2}, 1\right\}$ such that for all $0<\delta<\bar{\delta}$, the function

$$
P_{\delta}(y):=P(y)+\delta|y-x|^{2}-\delta^{2}
$$

satisfies

$$
P_{\delta}(x)=P(x)-\delta^{2}<u^{*}(x)
$$

and

$$
F\left[P_{\delta}\right](y) \in \mathcal{S}^{n \times n} \backslash \bar{U}, \quad \forall|y-x|<\delta^{1 / 9} .
$$

Clearly,

$$
P_{\delta}(y)>P(y), \quad \forall|y-x| \geq \delta^{1 / 5} .
$$


Define

$$
\hat{u}(y):= \begin{cases}\min \left\{u(y), P_{\delta}(y)\right\}, & \text { if }|y-x|<\delta^{1 / 5} \\ u(y), & \text { if }|y-x| \geq \delta^{1 / 5}\end{cases}
$$

By (67), $P_{\delta}$ is a supersolution of (9) in $\left\{y:|y-x|<\delta^{1 / 9}\right\}$. By (68), and using $P \geq u^{*} \geq u$, we have

$$
\hat{u}(y)=u(y)=\min \left\{u(y), P_{\delta}(y)\right\}, \quad \delta^{1 / 5} \leq|y-x| \leq \delta^{1 / 6} .
$$

It follows that $\hat{u}$, being the minimum of two supersolutions, is a supersolution of (9) in $\Omega$, and, because of the definition of $u$,

$$
u \leq \hat{u} \quad \text { in } \Omega .
$$

On the other hand we see from (66), the definition of $\hat{u}$ and (69) that there exists $\epsilon \in\left(0, \delta^{1 / 5}\right)$ such that

$$
u(y) \leq \hat{u}(y) \leq P_{\delta}(y)<u^{*}(x)-\epsilon, \quad \forall|y-x|<\epsilon .
$$

Thus

$$
u^{*}(x)=\lim _{r \rightarrow 0^{+}} \sup \{u(y)|| y-x \mid<r\} \leq u^{*}(x)-\epsilon,
$$

a contradition. The claim is proved, i.e. $u^{*}$ is a subsolution of (9) in $\Omega$.

Now we have proved that $u_{*}=u$ and $u^{*}$ are respectively supersolution and subsolution of (9) in $\Omega$, and $u_{*}=u^{*}$ on $\partial \Omega$. By the comparison principle Theorem 1.7(ii), $u^{*} \leq u$ in $\Omega$ and so $u=u_{*}=u^{*}$ is a solution of (9).

To conclude the section, let us remark that:

Remark 4.2. The conclusion of Theorem 1.5 is still valid for more general $(F, U)$ as in Theorem 1.7, or Theorem 3.2 or Theorem 3.3 provided that the function $L(x, s, p)$ is independent of $s$ and

$$
-\infty<\inf _{\bar{\Omega}} v \leq \sup _{\bar{\Omega}} w<+\infty
$$

\section{Lipschitz regularity of viscosity solutions}

In this section we prove Theorem 1.1, as an application of the comparison principle Theorem 1.7. We also consider some mild generalization regarding Lipschitz regularity of viscosity solutions for operator of the form (22). 
Proof of Theorem 1.1. Without loss of generality, we may assume that $\Omega=B(0,1)$ and we only need to prove that $u$ is Lipschitz continuous on $\overline{B\left(0, \frac{1}{2}\right)}$.

For any $x \in \overline{B\left(0, \frac{1}{2}\right)}, 0<\lambda \leq R:=\frac{1}{4}\left[\frac{\sup u\left(0, \frac{3}{4}\right)}{\inf _{B\left(0, \frac{3}{4}\right)} u}\right]^{-\frac{1}{n-2}}$, we define $u_{x, \lambda}$, the Kelvin transform of $u$, as

$$
u_{x, \lambda}(y):=\frac{\lambda^{n-2}}{|y-x|^{n-2}} u\left(x+\frac{\lambda^{2}(y-x)}{|y-x|^{2}}\right), \quad \forall y \in \overline{B\left(0, \frac{3}{4}\right) \backslash B(x, \lambda)} .
$$

For any $y \in \partial B\left(0, \frac{3}{4}\right)$, we have

$$
u_{x, \lambda}(y) \leq(4 R)^{n-2} \sup _{B\left(0, \frac{3}{4}\right)} u=\inf _{B\left(0, \frac{3}{4}\right)} u \leq u(y) .
$$

Also, we know that

$$
\lambda\left(A^{u_{x, \lambda}}\right) \in \partial \Gamma, \quad \text { in } B\left(0, \frac{3}{4}\right) \backslash \overline{B(x, \lambda)}, \quad \text { in the viscosity sense. }
$$

Since $u_{x, \lambda}=u$ on $\partial B(x, \lambda)$, by applying the comparison principle Theorem 1.7(b) with $\Omega=B\left(0, \frac{3}{4}\right) \backslash \overline{B(x, \lambda)}, U=\left\{M \in \mathcal{S}^{n \times n}: \lambda(M) \in \Gamma\right\}, F[\psi]=A[\psi], w=$ $-\frac{2}{n-2} \ln u_{x, \lambda}$ and $v=-\frac{2}{n-2} \ln u$, we have

$$
u_{x, \lambda} \leq u \text { in } B\left(0, \frac{3}{4}\right) \backslash \overline{B(x, \lambda)} \text { for any } 0<\lambda \leq R, x \in \overline{B\left(0, \frac{1}{2}\right)} .
$$

By [36, Lemma 2], (71) implies that $u$ is Lipschitz continuous on $\overline{B\left(0, \frac{1}{2}\right)}$. This concludes the proof.

As pointed out in the introduction, the above proof of Theorem 1.1 uses not only comparison principles but also conformal invariance property of the conformal Hessian. For general operators of the form (22), one does not expect a purely local regularity like that in Theorem 1.1 to hold, as illustrated by the following example.

Example 5.1. Let $U$ be the set of symmetric $n \times n$ matrices $M$ with $M_{11}>0$, and $L \equiv 0$. The equation $F[\psi] \in \partial U$ becomes

$$
\partial_{x_{1}}^{2} \psi=0
$$

Then, the comparison principle holds (by considering the restriction of $\psi$ to each line parallel to the $x_{1}$-axis). Nevertheless, for any continuous function $f: \mathbb{R}^{n-1} \rightarrow \mathbb{R}$, $\psi\left(x_{1}, x_{2}, \ldots, x_{n}\right)=f\left(x_{2}, \ldots, x_{n}\right)$ is a viscosity solution of $F[\psi] \in \partial U$, and clearly, the regularity of $\psi$ (with respect to the $x_{2}, \ldots, x_{n}$ variables) is not better than that of $f$. 
Despite the above negative example, by a variant of the proof of Theorem 1.1 using translational invariance rather than conformal invariance, we have the following partial generalization:

Corollary 5.2. Assume $n \geq 2$. Let $\Omega \subset \mathbb{R}^{n}$ be a bounded open set, $(F, U)$ be as in Theorem 1.7 with constant $\alpha$ and $\beta$. Assume that $\psi \in C^{0}(\Omega)$ is a viscosity solution to (9) in $\Omega$. If $\psi \in C^{0,1}(\overline{N \cap \Omega})$ for some open neighborhood of $\partial \Omega$, then $\psi \in C^{0,1}(\bar{\Omega})$ and

$$
\sup _{\Omega}|\nabla \psi| \leq \sup _{N \cap \Omega}|\nabla \psi|
$$

Before giving a proof, we remark that, in general, the Lipschitz regularity of $\psi$ on $\partial \Omega$ does not ensure that the solution $\psi$ is Lipschitz continuous in $\bar{\Omega}$.

Example 5.3. Consider the equation

$$
F[\psi]=\nabla^{2} \psi-|\nabla \psi|^{m} I \in \partial U
$$

where $m>2$ and $U$ is the set of symmetric $n \times n$ matrices with at least one positive eigenvalue. (This equation can be written equivalently as

$$
\operatorname{det}(F[\psi])=0 \text { and } F[\psi] \leq 0 .)
$$

Then $\psi(x)=-(m-1)^{\frac{m-2}{m-1}}(m-2)^{-1}(|x|-1)^{\frac{m-2}{m-1}}$ is a solution to (72) on $\Omega_{a}=\{1<$ $|x|<a\}$ for any $a>1$. Clearly $\psi$ is constant on each component of the boundary $\partial \Omega_{a}$, but $\psi \notin C^{0,1}\left(\overline{\Omega_{a}}\right)$.

Proof of Corollary 5.2. Shrinking $\Omega$ and $N$ if necessary, we may assume that $\psi \in$ $C^{0,1}(\bar{N})$.

We note that for any vector $e \in \mathbb{R}^{n}$ and any constant $c \in \mathbb{R}$, the function

$$
\psi_{e}(x):=\psi(x+e)
$$

satisfies $F\left[\psi_{e}+c\right] \in \partial U$ in $\Omega_{e}:=\{x: x+e \in \Omega\}$ in the viscosity sense. Thus, by the comparison principle Theorem [1.7(b),

$$
\psi \leq \psi_{e}+\max _{\partial\left(\Omega \cap \Omega_{e}\right)}\left(\psi-\psi_{e}\right) \text { in } \Omega \cap \Omega_{e} .
$$

In particular, there is some $\delta>0$ such that for $|e|<\delta$, we have $\partial\left(\Omega \cap \Omega_{e}\right) \subset \bar{N}$ and

$$
\psi \leq \psi_{e}+\sup _{N}|\nabla \psi \|| e \mid \text { in } \Omega \cap \Omega_{e}
$$

This implies the assertion.

Acknowledgments. Li is partially supported by NSF grant DMS-1501004. Wang is supported in part by the scholarship from China Scholarship Council under the Grant CSC No. 201406040131. 


\section{References}

[1] M. E. Amendola, G. Galise, And A. Vitolo, Riesz capacity, maximum principle, and removable sets of fully nonlinear second-order elliptic operators, Differential Integral Equations, 26 (2013), pp. 845-866.

[2] M. BARDI AND F. DA LIO, On the strong maximum principle for fully nonlinear degenerate elliptic equations, Arch. Math. (Basel), 73 (1999), pp. 276-285.

[3] H. Berestycki, I. Capuzzo Dolcetta, A. Porretta, and L. Rossi, Maximum principle and generalized principal eigenvalue for degenerate elliptic operators, J. Math. Pures Appl. (9), 103 (2015), pp. 1276-1293.

[4] I. Birindelli And F. Demengel, Comparison principle and Liouville type results for singular fully nonlinear operators, Ann. Fac. Sci. Toulouse Math. (6), 13 (2004), pp. 261-287.

[5] — Eigenvalue, maximum principle and regularity for fully non linear homogeneous operators, Commun. Pure Appl. Anal., 6 (2007), pp. 335-366.

[6] L. Caffarelli And X. Cabré, Fully nonlinear elliptic equations, vol. 43 of American Mathematical Society Colloquium Publications, American Mathematical Society, Providence, RI, 1995.

[7] L. Caffarelli, Y. Y. Li, And L. NirenberG, Some remarks on singular solutions of nonlinear elliptic equations III: viscosity solutions including parabolic operators, Comm. Pure Appl. Math., 66 (2013), pp. 109-143.

[8] L. Caffarelli, L. Nirenberg, and J. Spruck, The Dirichlet problem for nonlinear second-order elliptic equations. III. Functions of the eigenvalues of the Hessian, Acta Math., 155 (1985), pp. 261-301.

[9] S.-Y. A. Chang, M. J. Gursky, And P. YAng, An equation of MongeAmpère type in conformal geometry, and four-manifolds of positive Ricci curvature, Ann. of Math. (2), 155 (2002), pp. 709-787.

[10] S.-Y. S. CHEn, Local estimates for some fully nonlinear elliptic equations, Int. Math. Res. Not., (2005), pp. 3403-3425.

[11] M. G. Crandall, H. Ishit, And P.-L. Lions, User's guide to viscosity solutions of second order partial differential equations, Bull. Amer. Math. Soc. (N.S.), 27 (1992), pp. 1-67. 
[12] I. C. Dolcetta And A. Vitolo, On the maximum principle for viscosity solutions of fully nonlinear elliptic equations in general domains, Matematiche (Catania), 62 (2007), pp. 69-91.

[13] — - The weak maximum principle for degenerate elliptic operators in unbounded domains, (preprint).

[14] Y. Ge AND G. WAng, On a fully nonlinear Yamabe problem, Ann. Sci. École Norm. Sup. (4), 39 (2006), pp. 569-598.

[15] M. D. M. GonzÁlez, Singular sets of a class of locally conformally flat manifolds, Duke Math. J., 129 (2005), pp. 551-572.

[16] P. GuAn And G. WAng, Local estimates for a class of fully nonlinear equations arising from conformal geometry, Int. Math. Res. Not., (2003), pp. 14131432 .

[17] M. J. Gursky and J. A. Viaclovsky, Prescribing symmetric functions of the eigenvalues of the Ricci tensor, Ann. of Math. (2), 166 (2007), pp. 475-531.

[18] Z.-C. HAn, Y. Y. Li, And E. V. TeIXEIRA, Asymptotic behavior of solutions to the $\sigma_{k}$-Yamabe equation near isolated singularities, Invent. Math., 182 (2010), pp. 635-684.

[19] P. Hartman And L. NirenberG, On spherical image maps whose Jacobians do not change sign, Amer. J. Math., 81 (1959), pp. 901-920.

[20] F. R. Harvey And H. B. Lawson, JR., Existence, uniqueness and removable singularities for nonlinear partial differential equations in geometry, in Surveys in differential geometry. Geometry and topology, vol. 18 of Surv. Differ. Geom., Int. Press, Somerville, MA, 2013, pp. 103-156.

[21] H. IsHII, On uniqueness and existence of viscosity solutions of fully nonlinear second-order elliptic PDEs, Comm. Pure Appl. Math., 42 (1989), pp. 15-45.

[22] H. IshiI AND P.-L. LiOns, Viscosity solutions of fully nonlinear secondorder elliptic partial differential equations, J. Differential Equations, 83 (1990), pp. 26-78.

[23] R. JENSEN, The maximum principle for viscosity solutions of fully nonlinear second order partial differential equations, Arch. Rational Mech. Anal., 101 (1988), pp. 1-27. 
[24] Q. Jin, Local Hessian estimates for some conformally invariant fully nonlinear equations with boundary conditions, Differential and Integral Equations, 20 (2007), pp. 121-132.

[25] Q. Jin, A. LI, AND Y. Y. LI, Estimates and existence results for a fully nonlinear Yamabe problem on manifolds with boundary, Calc. Var. Partial Differential Equations, 28 (2007), pp. 509-543.

[26] B. Kawohl And N. Kutev, Strong maximum principle for semicontinuous viscosity solutions of nonlinear partial differential equations, Arch. Math. (Basel), 70 (1998), pp. 470-478.

[27] — Comparison principle and Lipschitz regularity for viscosity solutions of some classes of nonlinear partial differential equations, Funkcial. Ekvac., 43 (2000), pp. 241-253.

[28] _ Comparison principle for viscosity solutions of fully nonlinear, degenerate elliptic equations, Comm. Partial Differential Equations, 32 (2007), pp. 12091224 .

[29] S. KoIke And T. Kosugi, Remarks on the comparison principle for quasilinear PDE with no zeroth order terms, Commun. Pure Appl. Anal., 14 (2015), pp. 133-142.

[30] S. KoIKE AND O. LEY, Comparison principle for unbounded viscosity solutions of degenerate elliptic PDEs with gradient superlinear terms, J. Math. Anal. Appl., 381 (2011), pp. 110-120.

[31] A. Li AND Y. Y. Li, On some conformally invariant fully nonlinear equations, Comm. Pure Appl. Math., 56 (2003), pp. 1416-1464.

[32] — - On some conformally invariant fully nonlinear equations. II. Liouville, Harnack and Yamabe, Acta Math., 195 (2005), pp. 117-154.

[33] Y. Y. LI, Local gradient estimates of solutions to some conformally invariant fully nonlinear equations, Comm. Pure Appl. Math., 62 (2009), pp. 1293-1326. (C. R. Math. Acad. Sci. Paris 343 (2006), no. 4, 249-252).

[34] Y. Y. Li AND L. NGUYen, Harnack inequalities and Bôcher-type theorems for conformally invariant, fully nonlinear degenerate elliptic equations, Comm. Pure Appl. Math., 67 (2014), pp. 1843-1876. 
[35] — Symmetry, quantitative Liouville theorems and analysis of large solutions of conformally invariant fully nonlinear elliptic equations, (2016). http://arxiv.org/abs/1604.06039.

[36] — A fully nonlinear version of the Yamabe problem on locally conformally flat manifolds with umbilic boundary, (2009). http://arxiv.org/abs/0911.3366v1.

[37] Y. Y. Li and L. Nirenberg, A miscellany, in Percorsi incrociati (in ricordo di Vittorio Cafagna), Collana Scientifica di Ateneo, Universita di Salerno, 2010, pp. 193-208. http://arxiv.org/abs/0910.0323.

[38] N. Nadirashvili And S. Vlăduț, Singular solutions of conformal Hessian equation, (2014). http://arxiv.org/abs/1409.1454.

[39] W.-M. Sheng, N. S. Trudinger, And X.-J. Wang, The Yamabe problem for higher order curvatures, J. Differential Geom., 77 (2007), pp. 515-553.

[40] N. S. TRUdinger, Comparison principles and pointwise estimates for viscosity solutions of nonlinear elliptic equations, Rev. Mat. Iberoamericana, 4 (1988), pp. $453-468$.

[41] N. S. Trudinger And X.-J. WANG, On Harnack inequalities and singularities of admissible metrics in the Yamabe problem, Calc. Var. Partial Differential Equations, 35 (2009), pp. 317-338.

[42] J. A. Viaclovsky, Conformal geometry, contact geometry, and the calculus of variations, Duke Math. J., 101 (2000), pp. 283-316.

[43] _ Estimates and existence results for some fully nonlinear elliptic equations on Riemannian manifolds, Comm. Anal. Geom., 10 (2002), pp. 815-846.

[44] X.-J. WAng, A priori estimates and existence for a class of fully nonlinear elliptic equations in conformal geometry, Chinese Ann. Math. Ser. B, 27 (2006), pp. 169-178. 